\title{
Discontinuity in the asymptotic behavior of planar orthogonal polynomials under a perturbation of the Gaussian weight
}

\author{
Seung-Yeop Lee, Meng Yang
}

October 10, 2018

\begin{abstract}
We consider the orthogonal polynomials, $\left\{P_{n}(z)\right\}_{n=0,1, \ldots}$, with respect to the measure

$$
|z-a|^{2 c} e^{-N|z|^{2}} d A(z)
$$

supported over the whole complex plane, where $a>0, N>0$ and $c>-1$. We look at the scaling limit where $n$ and $N$ tend to infinity while keeping their ratio, $n / N$, fixed. The support of the limiting zero distribution is given in terms of certain "limiting potential-theoretic skeleton" of the unit disk. We show that, as we vary $c$, both the skeleton and the asymptotic distribution of the zeros behave discontinuously at $c=0$. The smooth interpolation of the discontinuity is obtained by the further scaling of $c=e^{-\eta N}$ in terms of the parameter $\eta \in[0, \infty)$.
\end{abstract}

\section{Introduction}

Consider the ensemble of $n$ point particles, $\left\{z_{j}\right\}_{j=1}^{n} \subset \mathbb{C}$, distributed according to the probability measure given by

$$
\frac{1}{\mathcal{Z}_{n}} \prod_{i<j}\left|z_{i}-z_{j}\right|^{2} \cdot \exp \left(-N \sum_{j=1}^{n} Q\left(z_{j}\right)\right) \cdot \prod_{j=1}^{n} d A\left(z_{j}\right)
$$

where $\mathcal{Z}_{n}$ is the normalization constant, $N>0$ is a (large) parameter, $Q: \mathbb{C} \rightarrow \mathbb{R} \cup\{\infty\}$ is called an external potential and $d A$ is the standard Lebesgue measure on the plane.

The statistical behavior of the particles has been studied [1] for a large class of potentials in various contexts including random normal matrices and two-dimensional Coulomb gas. For example, in the scaling limit where $n$ and $N$ tend to infinity while $n / N$ is fixed, it is known [12] that the counting measure of the particles converges weakly,

$$
\mathbb{E} \frac{1}{N} \sum_{j=1}^{n} \delta\left(z-z_{j}\right) \rightarrow \frac{\Delta Q}{4 \pi} \chi_{K}
$$


where $\Delta Q=\left(\partial_{x}^{2}+\partial_{y}^{2}\right) Q, \chi_{K}$ is the indicator function of the compact set $K \subset \mathbb{C}$ that we will call a droplet following [12], and the expectation value is taken with respect to the measure in (10).

A connection to orthogonal polynomials can be provided by Heine's formula. It says that the averaged characteristic polynomial of the $n$ particles is the (monic) orthogonal polynomial of degree $n$, i.e.,

$$
P_{n}(z)=P_{n, N}(z)=\mathbb{E} \prod_{j=1}^{n}\left(z-z_{j}\right)
$$

satisfies the orthogonality condition,

$$
\int_{\mathbb{C}} P_{n, N}(z) \overline{P_{m, N}(z)} e^{-N Q(z)} d A(z)=h_{n, N} \delta_{n m} \quad(n, m=0,1,2, \ldots),
$$

where $h_{n, N}$ is a (positive) norming constant. From this connection, one might wonder if the zero distribution of $P_{n}$ would tend to the averaged distribution of the particles. Though this is the case with the orthogonal polynomials on the real line (that corresponds to the particles confined on the line), in the cases of two-dimensional orthogonal polynomials so far studied [3, 2, 16, 14, 15, 5, the limiting zero distribution is observed to be concentrated on a small subset of the droplet, on some kind of potential-theoretic skeleton of $K 1$

A skeleton of $K$ will refer to a subset of (the polynomial hull of) $K$ with zero area, such that there exists a measure that is supported exactly on the skeleton and that generates the same logarithmic potential in the exterior of (the polynomial hull of) $K$ as the Lebesgue measure supported on $K$. One characteristic of such skeleton is that it can be discontinuous under the continuous variation of the droplet $K$. A simple example [10] comes from the sequence of polygons converging to a disk. The skeleton of the polygon, which is the set of rays connecting each vertex to the center, does not converge to the skeleton of the disk, the single point at the center. Such discontinuity can also occur, as we will see, when the perturbed droplets have real analytic boundary.

In this paper we ask whether the zero distribution of $P_{n}$ also exhibits the similar discontinuity under the variation of the underlying droplet or, equivalently, under the variation of the external potential. We consider the external potential given by

$$
Q(z)=|z|^{2}+\frac{2 c}{N} \log \frac{1}{|z-a|}, \quad c>-1, \quad a>0 .
$$

When $N$ is large and $c \ll N$, this represents a small perturbation of the Gaussian weight. It corresponds to the interacting Coulomb particles with charge +1 for each, in the presence of an extra particle with charge $+c$ at $a$. By a simple rotation of the plane, the above $Q$ covers the case with any nonzero $a \in \mathbb{C}$.

We are interested in the scaling limit where $N$ and $n$ go to infinity while the ratio, $n / N$, is fixed to a positive number. Below we will set $N=n$ without losing generality since the orthogonality (2) gives the relation

$$
P_{n, N}(z ; a)=\left(\frac{n}{N}\right)^{n / 2} P_{n, n}\left(\sqrt{\frac{N}{n}} z ; \sqrt{\frac{N}{n}} a\right),
$$

where $P_{n, N}(z ; a)=P_{n, N}(z)$ stands for the orthogonal polynomial with respect to the external potential given by (3). Though we will mostly use $N$, we will keep $n$ whenever the expression holds true for general $n \neq N$.

\footnotetext{
${ }^{1}$ In some cases, the skeleton is also called "mother body" [10, 11].
} 


\section{$1.1 \quad$ Limiting skeleton}

The potential (3) has been studied in [3] with the notation: $c_{\text {there }}=c_{\text {here }} / N$. Let us denote $c_{\text {there }}$ by $\gamma$ such that

$$
\gamma=\frac{c}{N} \text { and } Q(z)=|z|^{2}+2 \gamma \log \frac{1}{|z-a|} .
$$

To state Theorem 1 let us introduce $K_{\gamma}, \mu_{\gamma}$ and $\mathcal{S}_{\gamma}$, and define $\mu$ and $\mathcal{S}$.

Let $K_{\gamma} \subset \mathbb{C}$ be the compact set, called a droplet, so that

$$
\mu_{\gamma}^{(2 \mathrm{D})}=\frac{1}{4 \pi} \chi_{K_{\gamma}}
$$

is the unique probability measure that minimizes the energy functional,

$$
I[\mu]=\int Q d \mu+\frac{1}{2} \iint \log \frac{1}{|z-w|} d \mu(z) d \mu(w) .
$$

Let $\mathcal{S}_{\gamma}=\operatorname{supp} \mu_{\gamma}$ be the skeleton of $K_{\gamma}$, that is, the compact subset of $\mathbb{C}$ with zero area such that the probability measure $\mu_{\gamma}$ generates the same logarithmic potential as $\mu_{\gamma}^{(2 \mathrm{D})}$ :

$$
\left.U^{\mu_{\gamma}}(z)=U^{\mu_{\gamma}^{(2 \mathrm{D})}}(z), \quad z \notin \text { (polynomial convex hull of } K_{\gamma}\right) .
$$

Here we denote $U^{m}(z)=-\int \log |z-w| d m(w)$ for a positive Borel measure $m$. We note that such skeleton may not be unique in general. We give explicit definitions of $\mathcal{S}_{\gamma}$ and $\mu_{\gamma}$ in Section 2 .

We define the limiting skeleton $\mathcal{S}$ by

$$
\mathcal{S}=\{z \in \mathbb{C}: \operatorname{Re}(\log z-a z)=\log \beta-a \beta, \quad \operatorname{Re} z \leq \beta\},
$$

where

$$
\beta=\min \{a, 1 / a\} .
$$

From the equivalent representation of $\mathcal{S}$ in the real coordinates by

$$
\mathcal{S}=\left\{(x, y) \in \mathbb{R}^{2}: x^{2}+y^{2}=\beta^{2} e^{2 a(x-\beta)}, x \leq \beta\right\},
$$

it is a simple exercise to show that, $\mathcal{S} \subset \operatorname{clos} \mathbb{D}$ is a simple closed curve that encloses the origin and intersects $\beta$. We will denote the interior and the exterior of $\mathcal{S}$ by $\operatorname{Int} \mathcal{S}$ and Ext $\mathcal{S}$ respectively. See Figure 1 for some illustration of $\mathcal{S}$.

We define $\mu$ to be the probability measure supported on $\mathcal{S}$ given by

$$
d \mu(z)=\rho(z) d \ell(z)=\frac{1}{2 \pi}\left|a-\frac{1}{z}\right| d \ell(z), \quad z \in \mathcal{S},
$$

where $d \ell$ is the arclength measure of $\mathcal{S}$. Alternatively, the same measure can be written in terms of holomorphic differential by $d \mu(z)=(2 \pi \mathrm{i})^{-1}(1 / z-a) d z$.

Theorem 1. As $\gamma \rightarrow 0$ we have the convergences,

$$
K_{\gamma} \rightarrow \operatorname{clos} \mathbb{D}, \quad \mu_{\gamma} \rightarrow \mu, \quad \mathcal{S}_{\gamma} \rightarrow \mathcal{S},
$$

in the appropriate senses (i.e., respectively in Hausdorff metric, in weak-*, and in Hausdorff metric). 
The proof is in Section 2 ,

Remark 1. In both examples, the one by Gustafsson [10] and the one from the above theorem the discontinuity occurs when the droplet becomes a disk. It is an interesting question whether the discontinuity occurs with other shapes than disk. We think that, at least for an algebraic potential where the exterior of the droplet is a quadrature domain, the discontinuity happens only with the disk. This is for the simple reason that the disk is the only quadrature domain where the exterior domain is also a quadrature domain.

\subsection{Strong asymptotics of $P_{N}$ and the location of zeros}

Let us define

$$
\begin{aligned}
& \phi_{A}(z)=a(z-\beta)-\log \frac{z}{\beta}, \\
& \phi(z)= \begin{cases}\phi_{A}(z), & z \in \operatorname{Ext} \mathcal{S}, \\
-\phi_{A}(z), & z \in \operatorname{Int} \mathcal{S}\end{cases}
\end{aligned}
$$

Note that $\operatorname{Re} \phi \equiv 0$ on $\mathcal{S}$.

Let $U$ be a certain neighborhood of $\mathcal{S} \backslash\{\beta\}$ where $\operatorname{Re} \phi \leq 0$. See Figure 7 and the paragraph below Lemma 4 for more details. Let $D_{\beta}$ be a disk neighborhood of $\beta$ with a fixed radius such that the map $\zeta: D_{\beta} \rightarrow \mathbb{C}$ given below is univalent.

$$
\zeta(z)= \begin{cases}\sqrt{2 N \phi_{A}(z)}=a \sqrt{N}(z-\beta)(1+\mathcal{O}(z-\beta)) & \text { for } \quad a>1 \\ -N \phi_{A}(z)=\frac{1-a^{2}}{a} N(z-\beta)(1+\mathcal{O}(z-\beta)) & \text { for } \quad a<1\end{cases}
$$

Theorem 2. For $a>1$ and for any fixed nonzero $c>-1$, we have

$$
P_{N}(z)= \begin{cases}z^{N}\left(\frac{z}{z-\beta}\right)^{c}\left(1+\mathcal{O}\left(\frac{1}{N}\right)\right), & z \in \operatorname{Ext} \mathcal{S} \backslash\left(U \cup D_{\beta}\right), \\ -\frac{\beta^{N} \sqrt{2 \pi}\left(a^{2}-1\right)^{c}}{N^{1 / 2-c} a \Gamma(c)} \frac{e^{N a(z-\beta)}}{z-\beta}\left(\frac{z-\beta}{z-a}\right)^{c}\left(1+\mathcal{O}\left(\frac{1}{\sqrt{N}}\right)\right), & z \in \operatorname{Int} \mathcal{S} \backslash\left(U \cup D_{\beta}\right), \\ z^{N}\left(\frac{z}{z-\beta}\right)^{c}\left(1+\mathcal{O}\left(\frac{1}{N}\right)\right) & z \in U \backslash D_{\beta}, \\ -\frac{\beta^{N} \sqrt{2 \pi}\left(a^{2}-1\right)^{c}}{N^{1 / 2}-c} \frac{e^{N a(z-\beta)}}{z-\beta}\left(\frac{z-\beta}{z-a}\right)^{c}\left(1+\mathcal{O}\left(\frac{1}{\sqrt{N}}\right)\right), & \\ z^{N}\left(\left(\frac{z \zeta(z)}{z-\beta}\right)^{c} e^{\frac{\zeta^{2}(z)}{4}} D_{-c}(\zeta(z))+\mathcal{O}\left(\frac{1}{\sqrt{N}}\right)\right), & z \in D_{\beta} .\end{cases}
$$

Here $D_{-c}$ be the Parabolic cylinder function or Weber function and is defined by [19]

$$
D_{-c}(\zeta):=\frac{e^{\frac{\zeta^{2}}{4}}}{\mathrm{i} \sqrt{2 \pi}} \int_{\epsilon-\mathrm{i} \infty}^{\epsilon+\mathrm{i} \infty} e^{-\zeta s+\frac{s^{2}}{2}} s^{-c} d s, \quad \epsilon>0 .
$$


Theorem 3. For $a<1$ and for any fixed nonzero $c>-1$, we have

$$
P_{N}(z)=\left\{\begin{array}{cl}
z^{N}\left(\frac{z}{z-a}\right)^{c}\left(1+\mathcal{O}\left(\frac{1}{N^{\infty}}\right)\right), & z \in \operatorname{Ext} \mathcal{S} \backslash\left(U \cup D_{\beta}\right), \\
-\frac{a^{1+N}\left(1-a^{2}\right)^{c-1}}{N^{1-c} \Gamma(c)} \frac{e^{N a(z-a)}}{z-a}\left(1+\mathcal{O}\left(\frac{1}{N}\right)\right), & z \in \operatorname{Int} \mathcal{S} \backslash\left(U \cup D_{\beta}\right), \\
z^{N}\left(\frac{z}{z-a}\right)^{c}\left(1+\mathcal{O}\left(\frac{1}{N^{\infty}}\right)\right) & z \in U \backslash D_{\beta}, \\
-\frac{a^{1+N}\left(1-a^{2}\right)^{c-1}}{N^{1-c} \Gamma(c)} \frac{e^{N a(z-a)}}{z-a}\left(1+\mathcal{O}\left(\frac{1}{N}\right)\right), & \\
z^{N}\left(\frac{z}{z-a}\right)^{c}\left(1+\mathcal{O}\left(\frac{1}{N^{\infty}}\right)\right) & z \in D_{\beta} .
\end{array}\right.
$$

Here

$$
\hat{f}(\zeta)=\frac{-1}{2 \mathrm{i} \pi} \int_{\mathcal{L}} \frac{e^{s}}{s^{c}(s-\zeta)} d s,
$$

where the contour $\mathcal{L}$ begins at $-\infty$, circles the origin once in the counterclockwise direction, and returns to $-\infty$. The error bound $\mathcal{O}\left(1 / N^{\infty}\right)$ means $\mathcal{O}\left(1 / N^{k}\right)$ for arbitrary integer $k$.

One can check that the branch cut discontinuity of $(z /(z-a))^{c}$ in the last equation of (10) is canceled by the discontinuity of $\hat{f}$ so that the asymptotic expression of $P_{N}$ in $D_{\beta}$ is analytic.

From Theorem 2 and 3. one can notice that the zeros of $P_{N}$ can appear when the two terms in the asymptotic expressions of $P_{N}$ in $U \backslash D_{\beta}$ cancel each other and hence must have the same order in $N$. Such cancellation may be expressed in terms of $\phi_{A}$ as we presently explain below.

$$
\begin{array}{ll}
\left(\frac{z}{z-\beta}\right)^{c}=e^{N \phi_{A}(z)}\left(\frac{z-\beta}{z-a}\right)^{c} \frac{\sqrt{2 \pi}\left(a^{2}-1\right)^{c}}{a \Gamma(c) N^{\frac{1}{2}-c}(z-\beta)}, & \text { for } \quad a>1, \\
\left(\frac{z}{z-a}\right)^{c}=e^{N \phi_{A}(z)} \frac{a\left(1-a^{2}\right)^{c-1}}{N^{1-c} \Gamma(c)(z-a)}, & \text { for } \quad a<1 .
\end{array}
$$

Taking the logarithm of the absolute values on both sides and after simple calculations, we get

$$
\begin{array}{ll}
-\operatorname{Re} \phi_{A}(z)=\left(c-\frac{1}{2}\right) \frac{\log N}{N}-\frac{\log \Gamma(c)}{N}+\frac{1}{N} \log \left|\left(\frac{z-\beta}{z-a}\right)^{c} \frac{\sqrt{2 \pi}\left(a^{2}-1\right)^{c}}{a(z-\beta)^{1-c} z^{c}}\right|, & a>1, \\
-\operatorname{Re} \phi_{A}(z)=\frac{(c-1) \log N}{N}-\frac{\log \Gamma(c)}{N}+\frac{1}{N} \log \left|\frac{a\left(1-a^{2}\right)^{c-1}}{(z-a)^{1-c} z^{c}}\right|, & a<1 .
\end{array}
$$

As we will show in Lemma 4, $\operatorname{Re} \phi_{A}$ is positive (resp. negative) in $U \cap \operatorname{Int} \mathcal{S}$ (resp. in $U \cap \operatorname{Ext} \mathcal{S}$ ). For $a>1$, since the dominant term in the right hand side of (11) is $\left(c-\frac{1}{2}\right) \frac{\log N}{N}$, the zeros will approach $\mathcal{S}$ from Ext $\mathcal{S}$ for $c>\frac{1}{2}$ and from Int $\mathcal{S}$ for $c<\frac{1}{2}$. For $a<1$, since the dominant term in the right hand 

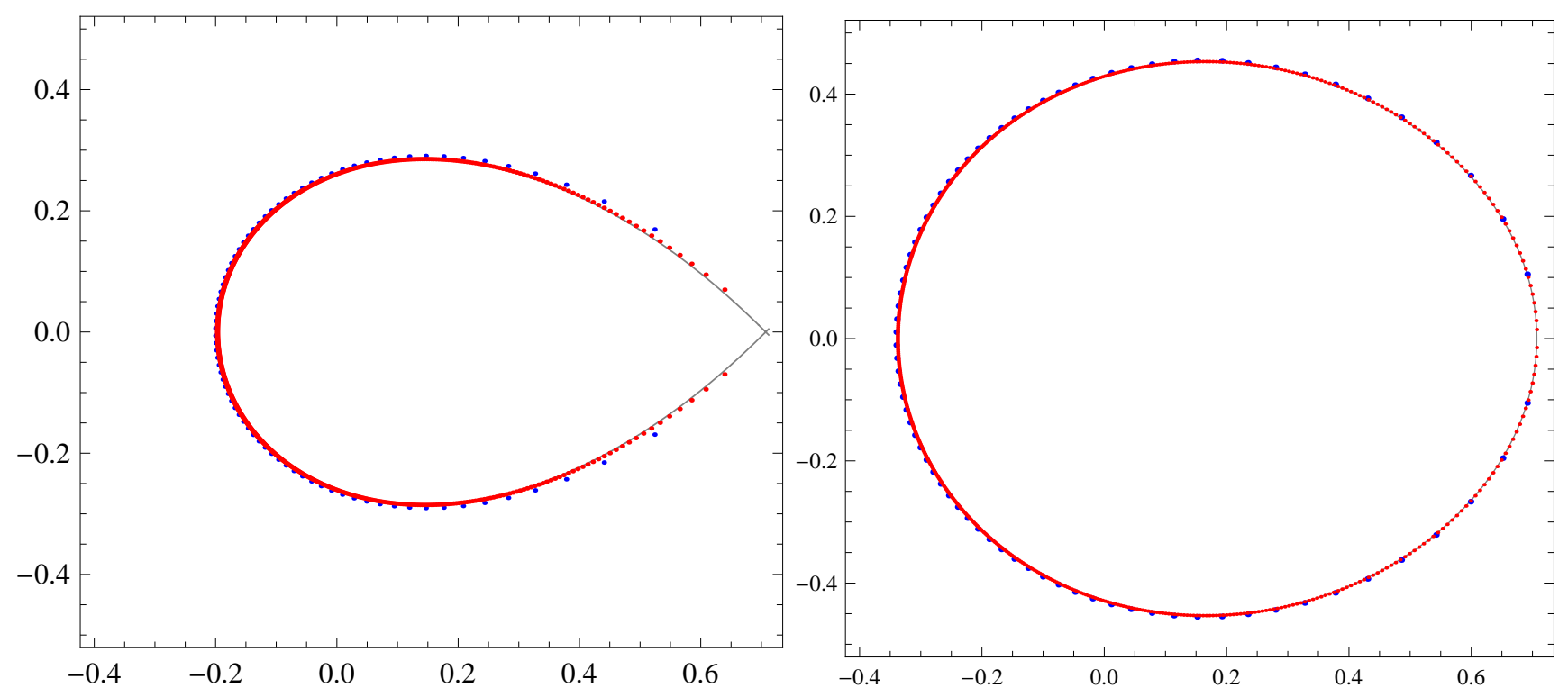

Figure 1: The zeros of orthogonal polynomials with degrees 80 (blue) and 600 (red) for $c=1$. The left is for $a=\sqrt{2}$ and the right is for $a=1 / \sqrt{2}$. In both cases, zeros are close to the curves representing $\mathcal{S}$.

side of (12) is $(c-1) \frac{\log N}{N}$, the zeros will approach $\mathcal{S}$ from Ext $\mathcal{S}$ for $c>1$ and from $\operatorname{Int} \mathcal{S}$ for $c<1$. See Figure 1. We also remark, without proof, that the limiting distribution of the zeros is given by $\mu$ which is explicitly given in (6). This can be proven, for example, using the method in [18] (Chapter III) and [17] (Theorem 2.3).

We remark that the case $-1<c<0$ is essentially treated in [2]. We note that the limiting locus of zeros remains the same for both the positive and negative $c$ (which seems unexpected according to Remark 1.2 in [2]). It turns out that, as the value of $c$ gets bigger, we need higher order corrections in the Riemann-Hilbert analysis. To obtain the result that works for an arbitrary value of $c$, therefore, we need an arbitrary order correction in the Riemann-Hilbert analysis. This is done in Section 5 using the method developed in [4].

We found that the limiting support of the zeros does not depend on $c$. Even for $c$ algebraically decaying in $N$ (e.g., $c=N^{-1000}$ ) the limiting support of the zeros converges to $\mathcal{S}$. However, when $c$ decays exponentially in $N$, say $c=e^{-\eta N}$, the right hand sides of both (11) and (12) converge to

$$
-\eta=-\lim _{N \rightarrow \infty} \frac{\log \Gamma\left(e^{-\eta N}\right)}{N}, \quad \eta>0
$$

and the zeros approach the curve in Int $\mathcal{S}$ given by the equation

$$
\operatorname{Re} \phi_{A}(z)=\eta \text {. }
$$

A similar "sensitive behavior of zeros under a parameter" has been observed in [13.

It is simple to observe that the family of curves given by (13) for $0 \leq \eta<\infty$ continuously interpolates between the curve $\mathcal{S}$ and the origin. In Figure 11, we show the curves satisfying (13) for $\eta=0.2$ and $\eta=0.4$, with the corresponding zeros. 

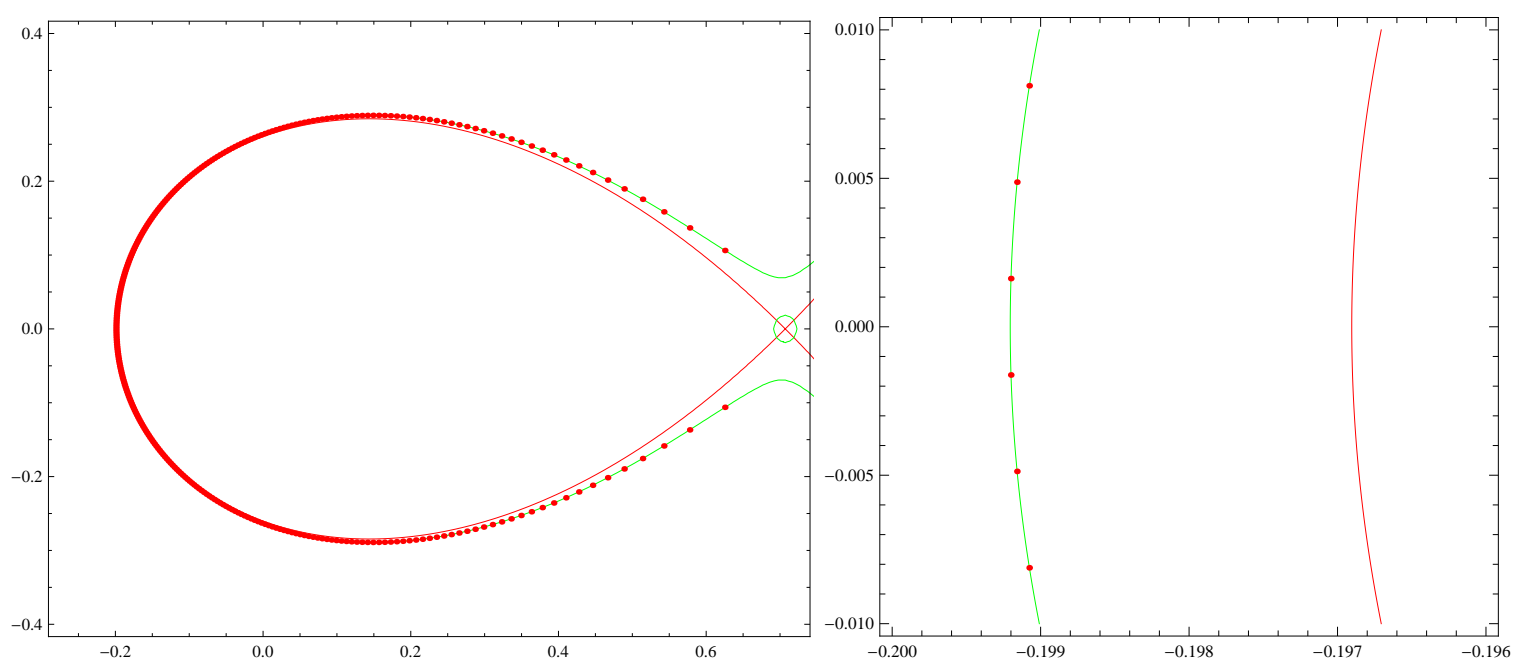

Figure 2: Zeros of orthogonal polynomials when $a=\sqrt{2}, c=1$ and $N=300$. The red line is $\mathcal{S}$ and the green line is the solution set of (11). The right figure is the enlarged view of the left figure.
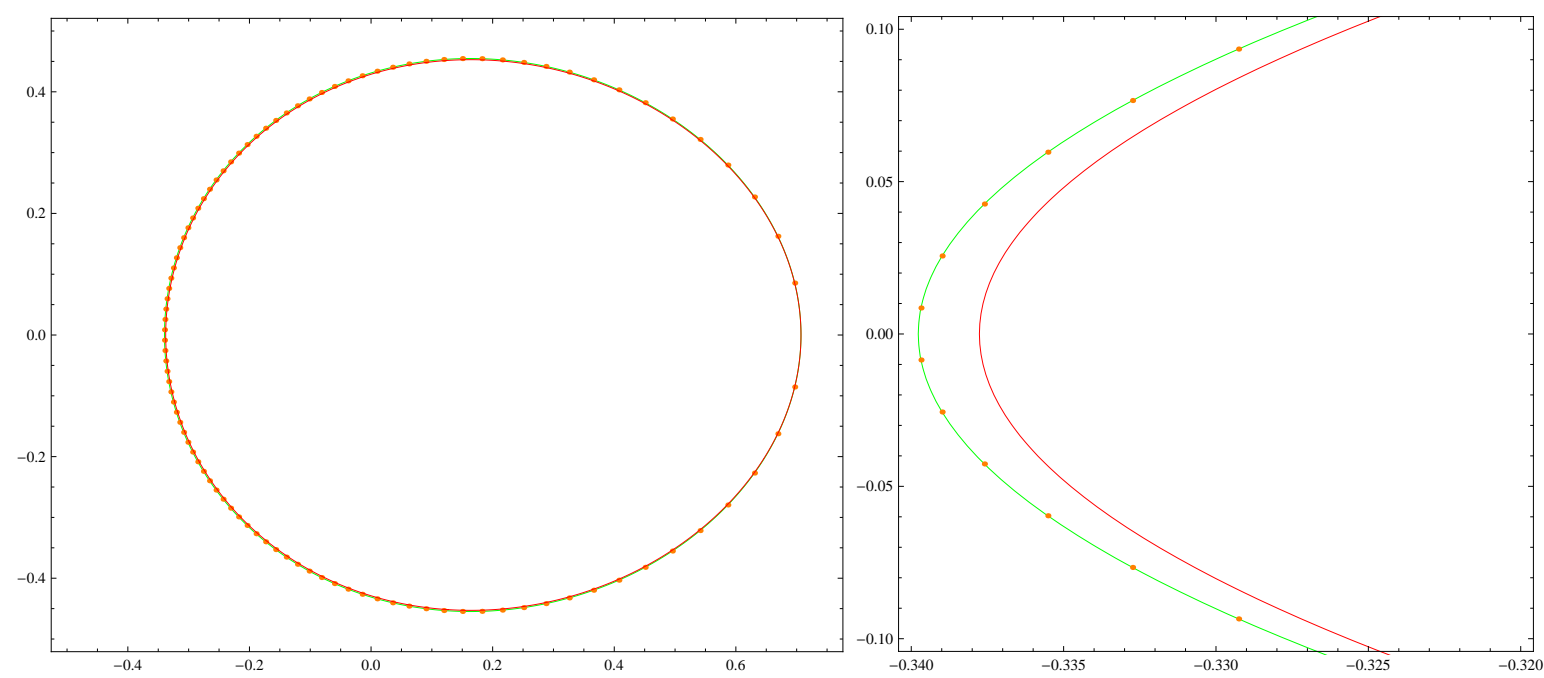

Figure 3: When $a=1 / \sqrt{2}, c=1$ and $N=100$. The red line is $\mathcal{S}$ and the green line is the solution set of (12). The right figure is the enlarged view of the left figure. 

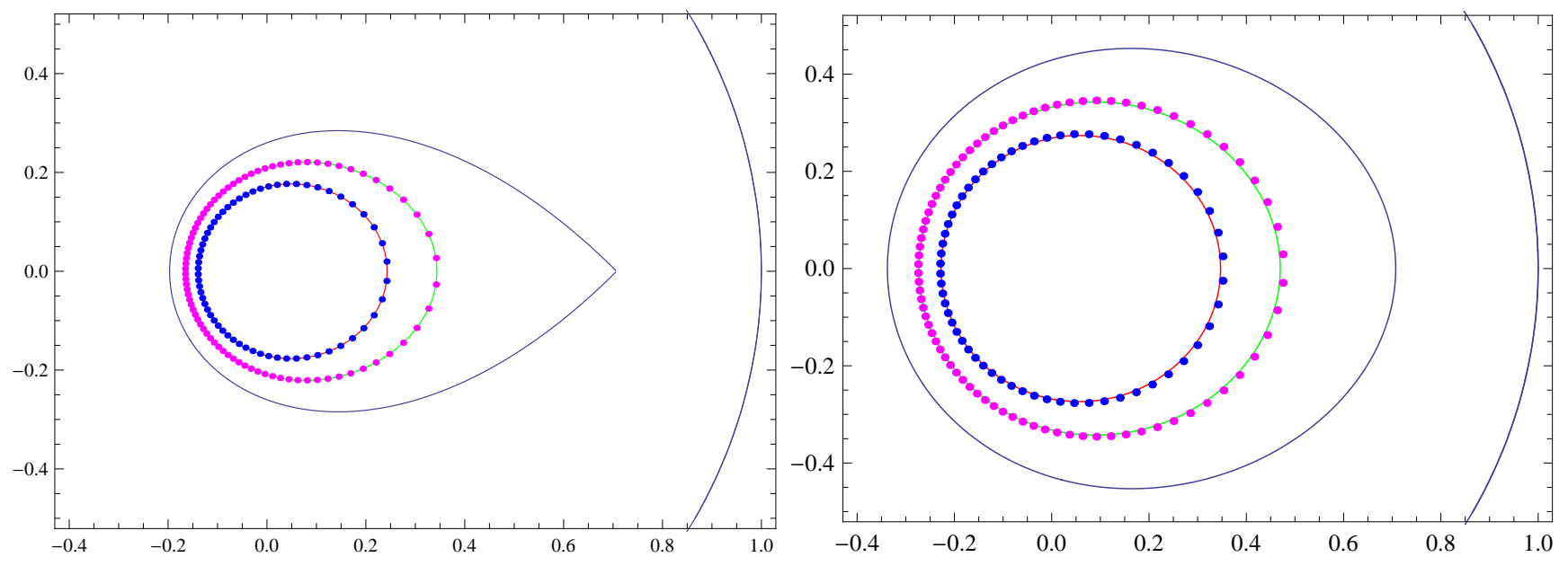

Figure 4: The zeros of orthogonal polynomials with degrees 60 (blue) and 80 (magenta) for $c=e^{-\eta n}$, where $\eta=0.4$ (blue) and $\eta=0.2$ (magenta). The left is for $a=\sqrt{2}$ and the right is for $a=1 / \sqrt{2}$. In both cases, zeros seem to converge to the curves given by (13) of the corresponding values.

To establish the behavior of zeros for scaling c, however, Theorem 2 and 3 are not enough as the error bounds in the theorems are for fixed $c$. For $c$ that scales to zero with $N$ we will prove Theorem 4 and 5 where the error bounds are uniform in $c$.

Remark 2. A simple way to understand the phenomenon is to recall the well-known instability of roots of polynomials, for example, the zeros of $P_{n}(z)=z^{n}+a / n^{k}$ still tend to the uniform distribution on the unit circle as $n \rightarrow \infty$ (for any fixed positive $k$ ) although the polynomial is a $\mathcal{O}\left(n^{-k}\right)$ perturbation of the monomial. This simple toy example already shows that a perturbation that interpolates between the two behaviors would require to have $a=e^{-n \eta}$. In this perspective it is not unexpected to see the exponentially small perturbations of the orthogonality measure in order to interpolate the behaviors.

Remark 3. A main message of the paper is that the asymptotic zero locus can be quite sensitive to the small perturbation of the underlying measure. In Figure 5 we give another numerical plot that supports such statement. The example considers the orthogonal polynomials with the cutoff. Though the cutoff may be considered as a "small perturbation" to the underlying Coulomb particle system, it seems to affect the polynomial significantly.

In the next section we prove Theorem 1 about the limiting skeleton. In section 3 we prove the asympototic result for $a>1$ and $c$ near 0 . In section 4 we prove the similar result for an arbitrary $c$. In section 5 we prove the asympototic result mostly following the arguments from the previous two sections. In the last section, we argue that the similar method will give the result for the critical case of $a \approx 1$, by showing that the local parametrix satisfies the Riemann-Hilbert problem for Painlevé IV equation.

Acknowledgement. The first author was supported by Simons Collaboration Grants for Mathematicians. We thank the referee for the insightful remarks - Remark 1 and 2 were prompted by the referee. 


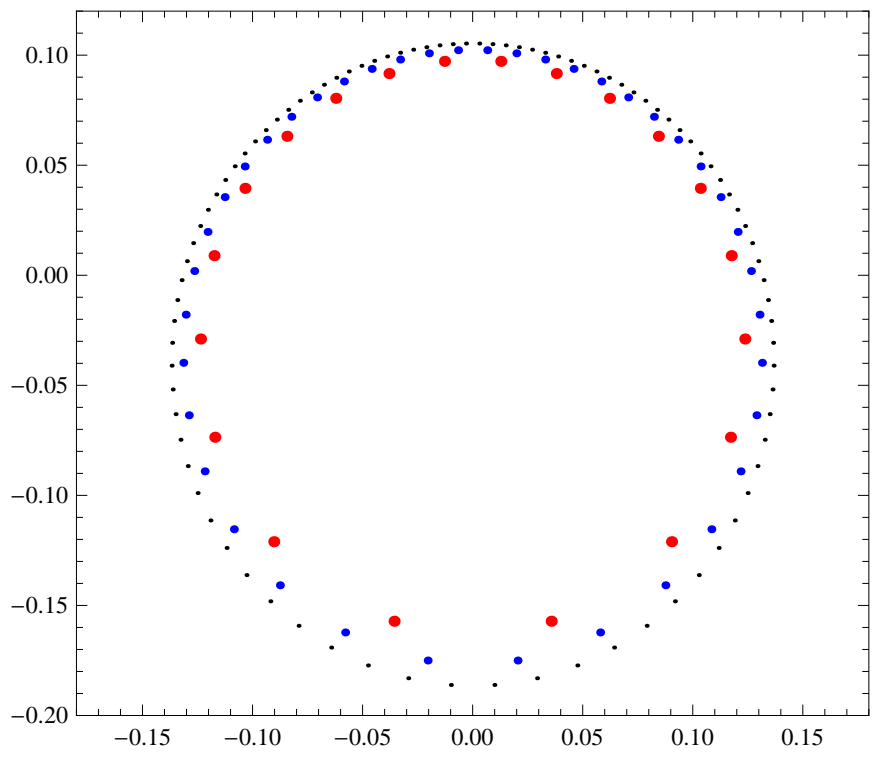

Figure 5: The zeros of orthogonal polynomials with degrees from $\{20,40,90\}$ and with the orthogonality measure given by $\chi_{K} \exp \left(-n|z|^{2}\right) d A(z)$ where $K=(-\infty,+\infty) \times[-3 \mathrm{i} / 2,+\mathrm{i} \infty) \subset \mathbb{C}$. The plot suggests that the limiting support of zeros is not the origin.

\section{The proof of Theorem 1}

For the convenience of the readers we reproduce the useful definitions in [3].

For $a<1$ for a sufficiently small $\gamma$ we define

$$
K_{\gamma}=\overline{D(0, \sqrt{1+\gamma})} \backslash D(a, \sqrt{\gamma}),
$$

where $D(a, r)$ stands for the disc with radius $r$ centered at $a$.

For $a<1$ we define $\mathcal{S}_{\gamma}$ to be the simple closed curve enclosing $[0, a]$ and intersecting

$$
\beta_{\gamma}=\frac{a^{2}+1-\sqrt{\left(1-a^{2}\right)^{2}-4 a^{2} \gamma}}{2 a}>a
$$

such that the quadratic differential $y_{\gamma}(z)^{2} d z^{2}$ is real and negative on $\mathcal{S}_{\gamma}$ where

$$
y_{\gamma}(z):=(-1)^{\chi_{\operatorname{Int} \mathcal{S}_{\gamma}}}\left[a+\frac{\gamma}{z-a}-\frac{1+\gamma}{z}\right] .
$$

Here, we denote the interior of the simple closed curve $\mathcal{S}_{\gamma}$ by $\operatorname{Int} \mathcal{S}_{\gamma}$. We recall that $\chi$ is the indicator function.

For $a \geq 1$, the set $K_{\gamma}$ is defined to be the closure of the interior of the real analytic Jordan curve given by the image of the unit circle under $f_{\gamma}$ given by

$$
f_{\gamma}(\nu)=\rho \nu-\frac{\kappa}{\nu-\alpha}-\frac{\kappa}{\alpha}
$$


whose parameters $\rho>0, \kappa \geq 0$, and $0<\alpha \leq 1 / a$ are given in terms of $a$ and $\gamma$ below. First, $\rho$ and $\kappa$ are given by

$$
\rho=\frac{1+a^{2} \alpha^{2}}{2 a \alpha}, \quad \kappa=\frac{\left(1-\alpha^{2}\right)\left(1-a^{2} \alpha^{2}\right)}{2 a \alpha} .
$$

The parameter $\alpha$ is given by the unique solution of $P_{\gamma}\left(\alpha^{2}\right)=0$ such that $0<\alpha \leq 1 / a$ where

$$
P_{\gamma}(X):=X^{3}-\left(\frac{a^{2}+4 \gamma+2}{2 a^{2}}\right) X^{2}+\frac{1}{2 a^{4}} .
$$

The uniqueness is easily seen by $P_{\gamma}(0)>0$ and $P_{\gamma}\left(1 / a^{2}\right)=-2 \gamma / a^{6}<0$. We note that, as $\gamma$ goes to zero, $\alpha$ goes to $1 / a, \kappa$ goes to zero and $\rho$ goes to 1 .

For $a \geq 1$ we define $\mathcal{S}_{\gamma}$ to be the smooth arc with the endpoints at

$$
\beta_{\gamma}:=\alpha \rho-\frac{\kappa}{\alpha}+2 \mathrm{i} \sqrt{\kappa \rho} \text { and } \overline{\beta_{\gamma}}
$$

such that the quadratic differential $y_{\gamma}(z)^{2} d z^{2}$ is real and negative on $\mathcal{S}_{\gamma}$ where

$$
y_{\gamma}(z):=\frac{a\left(z-b_{\gamma}\right) \sqrt{\left(z-\beta_{\gamma}\right)\left(z-\overline{\beta_{\gamma}}\right)}}{z(z-a)}, \quad b_{\gamma}=\frac{\rho}{\alpha} 2
$$

For all values of $a$, we define the probability measure $\mu_{\gamma}$ supported on $\mathcal{S}_{\gamma}$ by

$$
d \mu_{\gamma}=\frac{1}{2 \pi}\left|y_{\gamma}(z)\right| d \ell_{\gamma}
$$

where $d \ell_{\gamma}$ is the arclength measure of $\mathcal{S}_{\gamma}$.

For all values of $a$, we define $\phi_{\gamma}$ by

$$
\phi_{\gamma}(z)=\int_{\beta_{\gamma}}^{z} y_{\gamma}(s) d s
$$

where the integration contour lies in the simply connected domain $\mathbb{C} \backslash\left([0, \infty) \cup\left[\beta_{\gamma}, \overline{\beta_{\gamma}}\right]\right)$, where $\left[\beta_{\gamma}, \overline{\beta_{\gamma}}\right]$ stands for the vertical line segment connecting $\beta_{\gamma}$ and $\overline{\beta_{\gamma}}$ (for $a \geq 1,\left[\beta_{\gamma}, \overline{\beta_{\gamma}}\right]$ is a point on $\mathbb{R}^{+}$). One can consider $\phi_{\gamma}$ to be defined over the whole complex plane by analytic continuation over $[0, \infty) \cup\left[\beta_{\gamma}, \overline{\beta_{\gamma}}\right]$ consistently for all $\gamma$.

Lemma 1. As $\gamma$ goes to $0, \phi_{\gamma}$ converges to $\phi_{0}:=\phi_{\gamma=0}$ uniformly over a compact subset in $\mathbb{C} \backslash\{0, a\}$.

Proof. It is simple to check that, as $\gamma$ goes to zero, $\beta_{\gamma}$ converges to $\beta$ and $b_{\gamma}$ converges to $a$. Therefore $y_{\gamma}(z)$ converges to $y_{\gamma=0}(z)$, by choosing the branch cut of $y_{\gamma}$ at $\left[\beta_{\gamma}, \overline{\beta_{\gamma}}\right]$ that converges to $\beta$. This convergence is uniform away from the singularities of $y_{\gamma}$ at 0 and $a$.

Lemma 2. Let $I=\{\mathrm{i} t:-2 \pi \leq t \leq 0\}$. The mapping $\phi_{\gamma}: \mathcal{S}_{\gamma} \backslash\left\{\beta_{\gamma}, \overline{\beta_{\gamma}}\right\} \rightarrow I \backslash\{0,-\mathrm{i} 2 \pi\}$ is invertible.

\footnotetext{
${ }^{2} \operatorname{In}[3] b_{\gamma}$ is written as $\alpha / \rho$ by mistake.
} 
Proof. We prove this for $a>1$ as the other case is similiar. We get $\phi_{\gamma}\left(\beta_{\gamma}\right)=0$ by definition. We have

$$
\phi_{\gamma}\left(\overline{\beta_{\gamma}}\right)=\int_{\beta_{\gamma}}^{\overline{\beta_{\gamma}}} y_{\gamma}(s) d s=\frac{1}{2} \oint y_{\gamma}(s) d s,
$$

where, in the first integral, the integration contour can be taken along $\mathcal{S}_{\gamma}$ and, in the second integral, the integration contour goes around $\mathcal{S}_{\gamma}$ counterclockwise while the branch cut of $y_{\gamma}$ is placed at $\mathcal{S}_{\gamma}$ (instead of at $\left.\left[\beta_{\gamma}, \overline{\beta_{\gamma}}\right]\right)$. The latter integration contour can be deformed into three clockwise contours around $\infty, 0$ and $a$, which leads to

$$
\phi_{\gamma}\left(\overline{\beta_{\gamma}}\right)=-\frac{2 \pi \mathrm{i}}{2}\left(\operatorname{Res}_{z=\infty} y_{\gamma}(z)+\operatorname{Res}_{z=0} y_{\gamma}(z)+\operatorname{Res}_{z=a} y_{\gamma}(z)\right)
$$

By Lemma 2.19 in [3], we have $\operatorname{Res}_{z=\infty} y_{\gamma}(z)=1, \operatorname{Res}_{z=0} y_{\gamma}(z)=1+\gamma$, and $\operatorname{Res}_{z=a} y_{\gamma}(z)=-\gamma$ and, therefore, we have $\phi_{\gamma}\left(\overline{\beta_{\gamma}}\right)=-2 \pi$ i. Since $\phi_{\gamma}$ is continuous on $S_{\gamma}$ (here we again place the branch cut of $y_{\gamma}$ at $\left.\left[\beta_{\gamma}, \overline{\beta_{\gamma}}\right]\right)$ we have $I \subset \phi_{\gamma}\left(\mathcal{S}_{\gamma}\right)$. Since $\phi_{\gamma}$ has no critical point in $\mathcal{S}_{\gamma}$ except at the endpoints, $\phi_{\gamma}$ is 1-to-1 and $I=\phi_{\gamma}\left(\mathcal{S}_{\gamma}\right)$.

Lemma 3. Let $\left\{K_{j} \subset \mathbb{C}\right\}_{j=1}^{\infty}$ be bounded a sequence of compact sets such that $K_{\infty}$, the set of limit points of $\left\{K_{j}\right\}_{j=1}^{\infty}$, is also compact. If $K_{j}$ 's are all connected such that $b_{j} \in K_{j}$ and $\lim _{j \rightarrow \infty} b_{j}=b_{\infty}$ then $K_{\infty}$ is connected to $b_{\infty}$.

Proof. If not, there exist open sets $O_{1}$ and $O_{2}$ such that $K_{\infty}$ is the disjoint union of $K_{\infty} \bigcap O_{1}$ and $K_{\infty} \bigcap O_{2}$. Since $K_{\infty}$ is compact and since both $K_{\infty} \bigcap O_{1}$ and $K_{\infty} \bigcap O_{2}$ are closed in the relative topology of $K_{\infty}$, both $K_{\infty} \bigcap O_{1}$ and $K_{\infty} \bigcap O_{2}$ are compact and, therefore, there are disjoint open neighborhoods of the two disjoint compact sets (a property of a Hausdorff space). Without loss of generality, we can call the disjoint neighborhoods by $O_{1}$ and $O_{2}$. Suppose $b_{\infty} \in O_{2}$. For $j$ large enough we have $K_{j} \subset O_{1} \cup O_{2}$ and $b_{j} \in O_{2}$ and, therefore, $K_{j} \subset O_{2}$ because $K_{j}$ is connected. This is a contradiction.

Proof of Theorem 1. Assume $\mathcal{S}_{\gamma}$ does not converge to $\mathcal{S}$ in Hausdorff metric. Then there exist a sequence $\left\{p_{j}\right\} \subset \mathcal{S}$ and $\left\{\gamma_{j}\right\} \rightarrow 0$ such that $\operatorname{dist}\left(p_{j}, \mathcal{S}_{\gamma_{j}}\right)>2 \epsilon$ for some $\epsilon>0$. Taking a limit point $z \in \mathcal{S}$ of $\left\{p_{j}\right\}$ and choosing a subsequence if necessary we can assume $\operatorname{dist}\left(z, \mathcal{S}_{\gamma_{j}}\right)>\epsilon$ for all $j$ 's. Such $z$ cannot be $\beta \in \mathcal{S}$ because $\left\{\beta_{\gamma_{j}} \in \mathcal{S}_{\gamma_{j}}\right\}$ converges to $\beta$ as $j$ goes to $\infty$. Since $\phi_{\gamma_{j}}: \mathcal{S}_{\gamma_{j}} \backslash\left\{\beta_{\gamma_{j}}, \overline{\beta_{\gamma_{j}}}\right\} \rightarrow I \backslash\{0,-2 \pi \mathrm{i}\}$ is invertible by Lemma 2 , we can define

$$
z_{j}:=\phi_{\gamma_{j}}^{-1} \circ \phi_{0}(z) \in \mathcal{S}_{\gamma_{j}}
$$

Let $z_{\infty}$ be a limit point of $\left\{z_{j}\right\}$, then $z_{\infty} \notin\{0, a\}$ because $\mathcal{S}_{\gamma_{j}}$ is uniformly away from 0 and $a$ for sufficiently small $\gamma_{j}$. We also have $z_{\infty} \neq \beta$ (and similarly, $z_{\infty} \neq \bar{\beta}$ ) because, if not, $\left|z_{j}-\beta_{\gamma_{j}}\right|$ would go to zero while $\left|\phi_{\gamma_{j}}\left(z_{j}\right)-\phi_{\gamma_{j}}\left(\beta_{\gamma_{j}}\right)\right|=\left|\phi_{0}(z)\right|>0$.

Since $\left(\operatorname{clos}\left\{z_{j}\right\}\right) \cap\{0, a\}=\emptyset$ Lemma 1 says that

$$
\left|\phi_{0}(z)-\phi_{0}\left(z_{j}\right)\right|=\left|\phi_{\gamma_{j}}\left(z_{j}\right)-\phi_{0}\left(z_{j}\right)\right| \stackrel{j \rightarrow \infty}{\longrightarrow} 0 .
$$

Since a subsequence of $\left\{\phi_{0}\left(z_{j}\right)\right\}$ converges to $\phi_{0}\left(z_{\infty}\right)$ by the continuity of $\phi_{0}$, we have

$$
\phi_{0}(z)=\phi_{0}\left(z_{\infty}\right)
$$



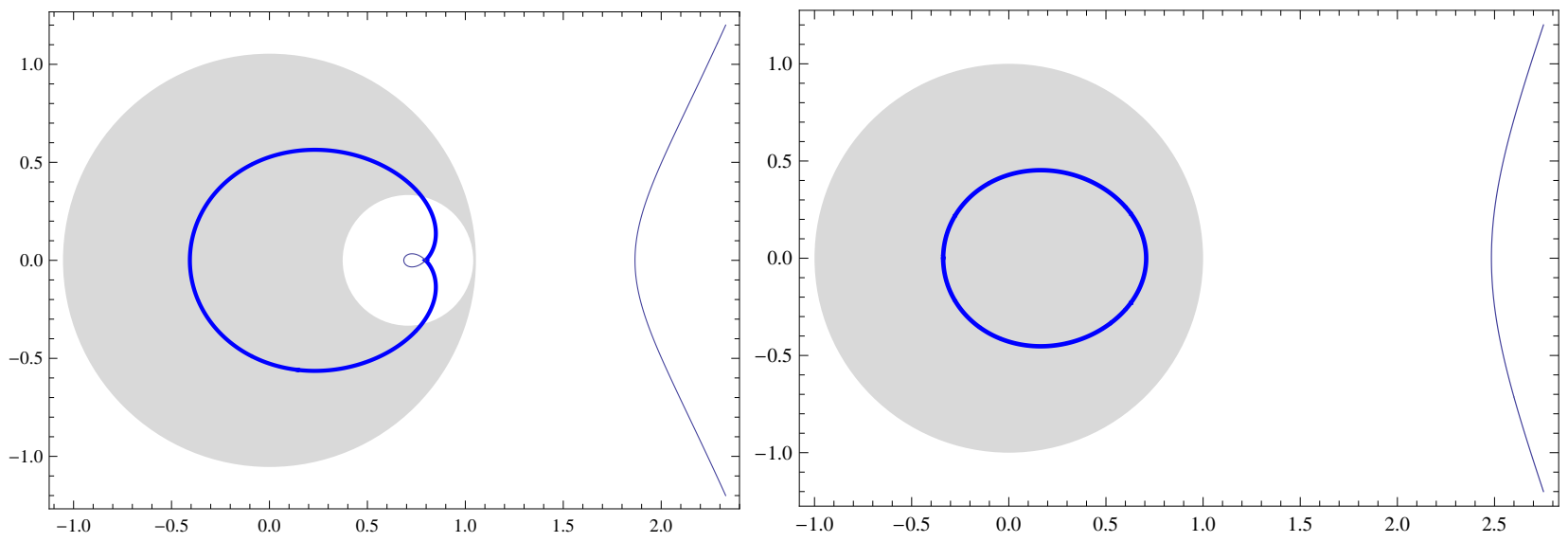

Figure 6: Illustration of the convergence, $\mathcal{S}_{\gamma} \rightarrow \mathcal{S}$ and $K_{\gamma} \rightarrow K$, when $a=1 / \sqrt{2}$. For $\gamma=1 / 9$ (left), $\mathcal{S}_{\gamma}$ is drawn with thick line and the rest of the set $\left\{z: \operatorname{Re} \phi_{\gamma}(z)=0\right\}$ is drawn with the thin line; $K$ is the shaded region. Same for $\gamma=0$ (right).

Let $\mathcal{S}_{\infty}$ be the set of limit points of $\left\{\mathcal{S}_{\gamma_{j}}\right\}$. By Lemma $3 \mathcal{S}_{\infty}$ is connected to $\beta$. Since $\mathcal{S}$ is the only component of $\phi_{0}^{-1}(I)$ that is connected to $\beta$ we have $\mathcal{S}_{\infty} \subset \mathcal{S}$. From (15) and $z_{\infty} \in \mathcal{S} \backslash\{\beta, \bar{\beta}\}$, we get $z=z_{\infty}$ by Lemma 2. This is a contradiction because $z_{\infty}$ is a limit point of $\left\{\mathcal{S}_{\gamma_{j}}\right\}$ and, therefore, $\operatorname{dist}\left(z, z_{\infty}\right) \geq \epsilon$. This concludes the proof of $\mathcal{S}_{\gamma} \rightarrow \mathcal{S}$.

For $a<1$, the convergence of $K_{\gamma}$ to clos $\mathbb{D}$ follows from (14).

For $a \geq 1$, we need to show that $\partial K_{\gamma}=f_{\gamma}(\partial \mathbb{D})$ converges to $\partial \mathbb{D}$. Recall that, as $\gamma$ goes to zero, $\alpha$ goes to $1 / a, \kappa$ goes to zero and $\rho$ goes to 1 . It follows that $\lim _{\gamma \rightarrow 0} f_{\gamma}(v)=v$, which means $K_{\gamma} \rightarrow$ clos $\mathbb{D}$.

For all $a$, the convergence of $\mu_{\gamma}$ to $\mu$ follows from the facts $\mathcal{S}_{\gamma} \rightarrow \mathcal{S}$ and $\lim _{\gamma \rightarrow 0}\left|y_{\gamma}(z)\right|=2 \pi \rho(z)$ where $\rho$ is defined in (6).

\section{Matrix Riemann-Hilbert Problem}

The following fact is from [3]:

Theorem. Let $\Gamma$ be a simple closed curve enclosing the line segment $[0, a] \subset \mathbb{C}$ and oriented counterclockwise. Let the analytic function $\omega_{n, N}$ on $\mathbb{C} \backslash[0, a]$ be defined by

$$
\omega_{n, N}(z):=\left(\frac{z-a}{z}\right)^{c} \frac{e^{-N a z}}{z^{n}}
$$


where we choose the principal branch. Then the Riemann-Hilbert problem,

$$
\begin{cases}Y(z) \text { is holomorphic in } \mathbb{C} \backslash \Gamma, & \\
Y_{+}(z)=Y_{-}(z)\left[\begin{array}{cc}
1 & \omega_{n, N}(z) \\
0 & 1
\end{array}\right], & z \in \Gamma \\
Y(z)=\left(I+\mathcal{O}\left(\frac{1}{z}\right)\right)\left[\begin{array}{cc}
z^{n} & 0 \\
0 & z^{-n}
\end{array}\right], & z \rightarrow \infty\end{cases}
$$

has the unique solution given by

$$
Y(z)=\left[\begin{array}{cc}
P_{n}(z) & \frac{1}{2 \pi \mathrm{i}} \int_{\Gamma} \frac{P_{n}(w) \omega_{n, N}(w)}{w-z} d w \\
Q_{n-1}(z) & \frac{1}{2 \pi \mathrm{i}} \int_{\Gamma} \frac{Q_{n-1}(w) \omega_{n, N}(w)}{w-z} d w
\end{array}\right]
$$

where $Q_{n-1}(z)$ is the unique polynomial of degree $n-1$ such that

$$
\frac{1}{2 \pi \mathrm{i}} \int_{\Gamma} \frac{Q_{n-1}(w) \omega_{n, N}(w)}{w-z} d w=\frac{1}{z^{n}}\left(1+\mathcal{O}\left(\frac{1}{z}\right)\right) .
$$

Lemma 4. For $a<1$, there exists a neighborhood $V$ of $\overline{\operatorname{Int} \mathcal{S}}$ such that $\operatorname{Re} \phi(z)<0$ on $V \backslash \mathcal{S}$ and the boundary of $V$ is a smooth Jordan curve. For $a \geq 1$, there exists a domain $V$ such that it contains $\overline{\operatorname{Int} \mathcal{S}} \backslash\{\beta\}$ and its boundary, $\partial V$, is a smooth Jordan curve that intersects $\beta$. Also $\mathcal{S}$ is smooth except at $\beta$, where it makes a corner with the inner (i.e. towards $\operatorname{Int} \mathcal{S}$ ) angle $\pi / 2$. Lastly, $\operatorname{Re} \phi>0$ on $(\beta, a]$.

Proof. From the definition (7) of $\phi, \operatorname{Re} \phi$ is harmonic function away from $\mathcal{S}$ and the origin. Since $\operatorname{Re} \phi(z)$ diverges to $-\infty$ as $z$ goes to $0, \operatorname{Re} \phi(z)$ has to be negative everywhere in $\operatorname{Int} \mathcal{S}$ - otherwise $\operatorname{Re} \phi(z)$ has a local maximum in Int $\mathcal{S}$, which is impossible. For $a<1$, since the only critical point, $1 / a$, of $\phi$ is away from $\mathcal{S}$ and since $\operatorname{Re} \phi_{A}$ is harmonic in a neighborhood of $\mathcal{S}, \operatorname{Re} \phi$ is negative in the vicinity of $\mathcal{S}$. For $a \geq 1$, since $\beta$ is the only critical point of $\phi_{A}$, the claim in the lemma about the local shape of $\mathcal{S}$ near $\beta$ and about $\partial V$ being intersecting $\beta$ follows by the local analysis of the harmonic function $\operatorname{Re} \phi_{A}(z)$. Specifically, $\operatorname{Re} \phi_{A}(z)$ is positive along the real axis on $(0, \infty) \backslash\{\beta\}$, and is negative near $\beta$ in the vertical direction (i.e. imaginary direction) from $\beta$.

Using $V$ from the above lemma, we define the domain $U$ as below

$$
U=V \backslash \overline{V_{0}}
$$

Here $V_{0}$ is a small open neighborhood of $[0, \beta]$ such that its boundary, $\partial V_{0}$, is a smooth Jordan curve that is arbitrarily close to $[0, \beta]$, see Figure [7. The region $U$ is the simply-connected (when $a \geq 1$ ) or doubly-connected (when $a<1$ ) open neighborhood of $\mathcal{S} \backslash \overline{V_{0}}$, disjoint from $[0, a]$ and with a (piecewise) smooth boundary. We assign the counterclockwise orientation on $\partial U \cap \operatorname{Ext} \mathcal{S}$ with respect to the domain $U$ and the counterclockwise orientation on $\partial U \cap \operatorname{Int} \mathcal{S}$ with respect to $V_{0}$.

From now on we let $\Gamma$ exactly match $\mathcal{S}$ inside $U$ and away from a small neighborhood of $\beta$. When $a>1$, a part of the contour $\Gamma$ goes outside $U$ around the line segment $[\beta, a]$, see Figure 8 . Near $\beta$ the 

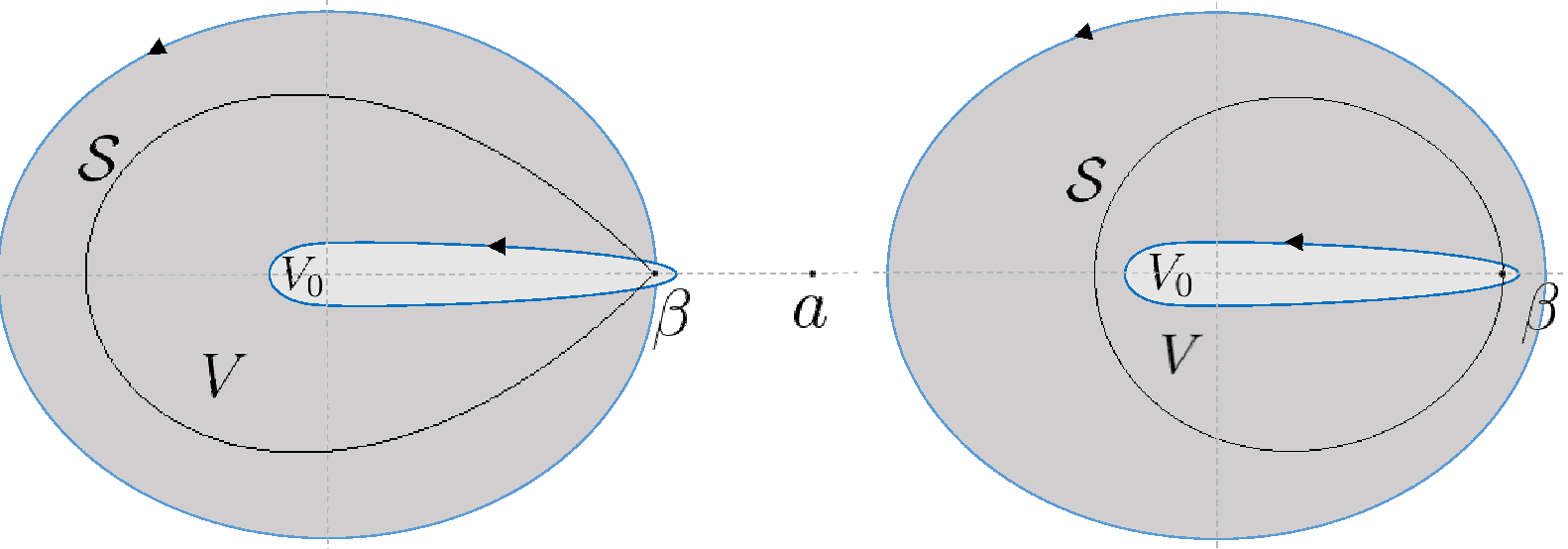

Figure 7: $\quad V$ and $V_{0}$ for $a>1$ (left) and $a<1$ (right), $\mathcal{S}$ is the black curve, $V$ is the interior of the contour enclosing the shaded region, $V_{0}$ is the interior of the contour enclosing the non-shaded region. These domains are used to define the domain $U$ at (16).
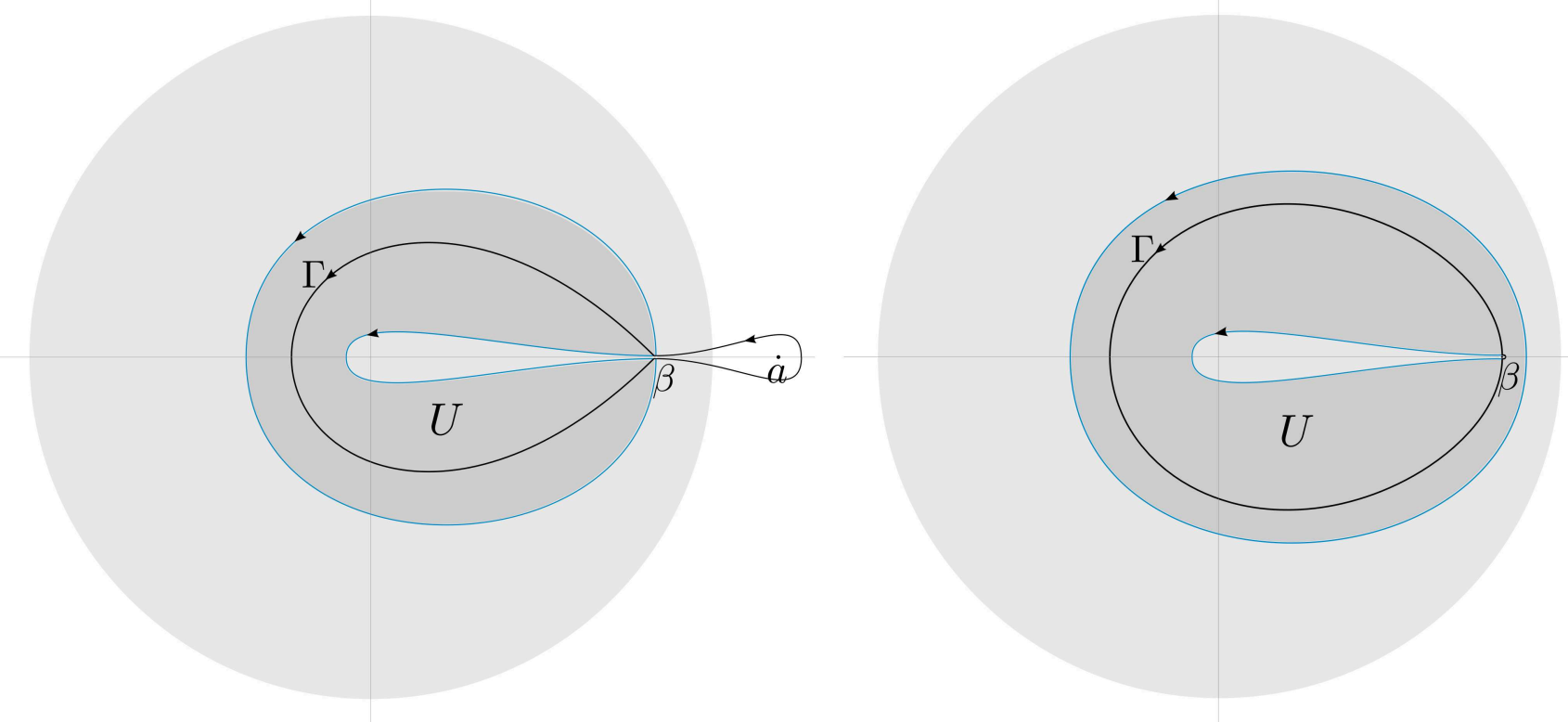

Figure 8: Contours for the Riemann-Hilbert problem of $\Phi$ when $a>1$ (left) and $a<1$ (right). $\Gamma$ is the black curves and $U$ is the shaded region bounded by the blue curves. 
reader should not be concerned too much about the exact arrangement of $\Gamma$ and $U$ as it will become clear when we define the local parametrix.

Below we define the complex logarithmic potential of $\mu$ (6) by

$$
g(z)=\int \log (z-w) d \mu(w)
$$

where the specific branch of the $\log$ is chosen below. As a function of $z$, this equals $\log z$ (modulo $2 \pi \mathrm{i}$ ) when $z \in \operatorname{Ext} \mathcal{S}$ by (4) and Theorem 1, and has continuous real part, since the jump of $g$ on $\mathcal{S}$ is purely imaginary. These properties and (5) determine the explicit expression of this function as follows,

$$
g(z)= \begin{cases}\log z, & z \in \overline{\operatorname{Ext} \mathcal{S}} \\ a z+\log \beta-a \beta, & z \in \operatorname{Int} \mathcal{S} .\end{cases}
$$

From the $g$-function above, we can write

$$
\phi(z)=a z+\log z-2 g(z)+\ell, \quad \ell=\log \beta-a \beta
$$

so that $\operatorname{Re} \phi(z)=0$ when $z \in \mathcal{S}$.

Following the standard nonlinear steepest descent method [7, 8] applied to the matrix Riemann-Hilbert problem for $Y$, we define $Z$ as the final object after the multiple transforms of $Y$ given by

$$
Z(z)=e^{\frac{-N \ell}{2} \sigma_{3}} Y(z) e^{-N g(z) \sigma_{3}} e^{\frac{N \ell}{2} \sigma_{3}}\left[\star\left(\frac{z}{z-a}\right)^{c} e^{N \phi(z)} \quad 1\right],
$$

where

$$
\star= \begin{cases}1, & \text { when } z \in U \cap \operatorname{Ext} \Gamma, \\ -1, & \text { when } z \in U \cap \operatorname{Int} \Gamma, \\ 0, & \text { when } z \notin U .\end{cases}
$$

Then $Z$ solves the following Riemann-Hilbert problem,

$$
\begin{cases}Z_{+}(z)=Z_{-}(z)\left[\begin{array}{cc}
1 & 0 \\
\left(\frac{z}{z-a}\right)^{c} e^{N \phi(z)} & 1
\end{array}\right], & z \in \partial U, \\
Z_{+}(z)=Z_{-}(z)\left[\begin{array}{cc}
0 & \left(\frac{z-a}{z}\right)^{c} \\
-\left(\frac{z}{z-a}\right)^{c} & 0
\end{array}\right], & z \in \Gamma \cap U, \\
Z_{+}(z)=Z_{-}(z)\left[\begin{array}{cc}
1 & \left(\frac{z-a}{z}\right)^{c} e^{-N \phi(z)} \\
0 & 1
\end{array}\right], & z \in \Gamma \backslash U . \\
Z(z)=I+\mathcal{O}\left(z^{-1}\right), & z \rightarrow \infty .\end{cases}
$$

We define

$$
\Phi(z)=\left\{\begin{array}{lc}
{\left[\begin{array}{cc}
\left(\frac{z}{z-\beta}\right)^{c} & 0 \\
0 & \left(\frac{z-\beta}{z}\right)^{c}
\end{array}\right],} & z \in \operatorname{Ext} \Gamma, \\
& {\left[\begin{array}{cc}
0 & \left(\frac{z-a}{z-\beta}\right)^{c} \\
-\left(\frac{z-\beta}{z-a}\right)^{c} & 0
\end{array}\right], \quad z \in \operatorname{Int} \Gamma,}
\end{array}\right.
$$


that satisfies the Riemann-Hilbert problem,

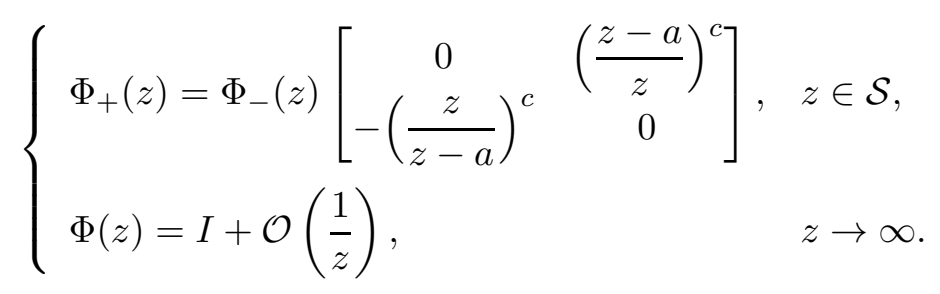

Note that, when $a \leq 1$ and $z \in \operatorname{Int} \mathcal{S}$ we have $\Phi(z)=\left[\begin{array}{cc}0 & 1 \\ -1 & 0\end{array}\right]$. Also note that $\Phi$ is not the only solution to the above Riemann-Hilbert problem - for any rational matrix function $\mathcal{R}(z)$ with a pole at $\beta$ such that $\mathcal{R}(\infty)=I, \mathcal{R}(z) \Phi(z)$ is a solution. We will use this fact in the next section.

\section{$4 \quad a>1$ : when $c$ near 0}

From the definition of $\phi_{A}$ at (7), we obtain

$$
\phi_{A}(z)=\frac{a^{2}}{2}(z-\beta)^{2}(1+\mathcal{O}(z-\beta)) .
$$

Let $D_{\beta}$ be a disk centered at $\beta$ such that there exists a univalent map $\zeta: D_{\beta} \rightarrow \mathbb{C}$ as defined in (8) ). Under the mapping $\zeta$ the contour $\mathcal{S}$ maps into $\left[0, e^{3 \pi \mathrm{i} / 4} t\right] \cup\left[0, e^{-3 \pi \mathrm{i} / 4} t\right]_{t \in[0, \infty)}$.

In this section we intend to find $\mathcal{P}: D_{\beta} \rightarrow \mathbb{C}^{2 \times 2}$ such that

$$
Z^{\infty}(z)=\Phi(z)\left(\frac{z-a}{z}\right)^{\frac{c}{2} \sigma_{3}} \mathcal{P}(z)\left(\frac{z-a}{z}\right)^{-\frac{c}{2} \sigma_{3}}, \quad z \in D_{\beta}
$$

satisfies the jump condition of $Z$ at (18), i.e., we require $\mathcal{P}$ to satisfy, in $D_{\beta}$,

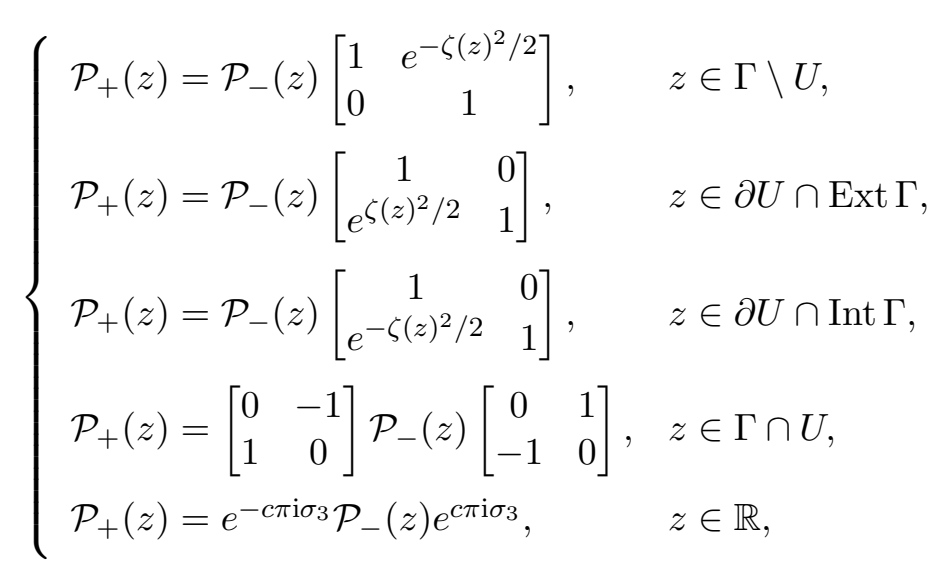

and the boundary condition, $\mathcal{P}(z) \sim I$ on $\partial D_{\beta}$. The fourth equation of (20) comes from $\Phi$ in (19) and the last equation comes from the (conjugating) factors $((z-a) / z)^{ \pm(c / 2) \sigma_{3}}$ in (19). The jump contours, $\Gamma \backslash U$ and $\partial U \cap \operatorname{Int} \Gamma$, can be pushed arbitrarily close to the real axis, so that the jump contours of $\mathcal{P}$ consists of $\mathbb{R}, i \mathbb{R}$ and $\left\{t e^{ \pm \mathrm{i} 3 \pi / 4}\right\}_{0<t<\infty}$. See Figure 9 for the illustration of the jump contours in $D_{\beta}$. 

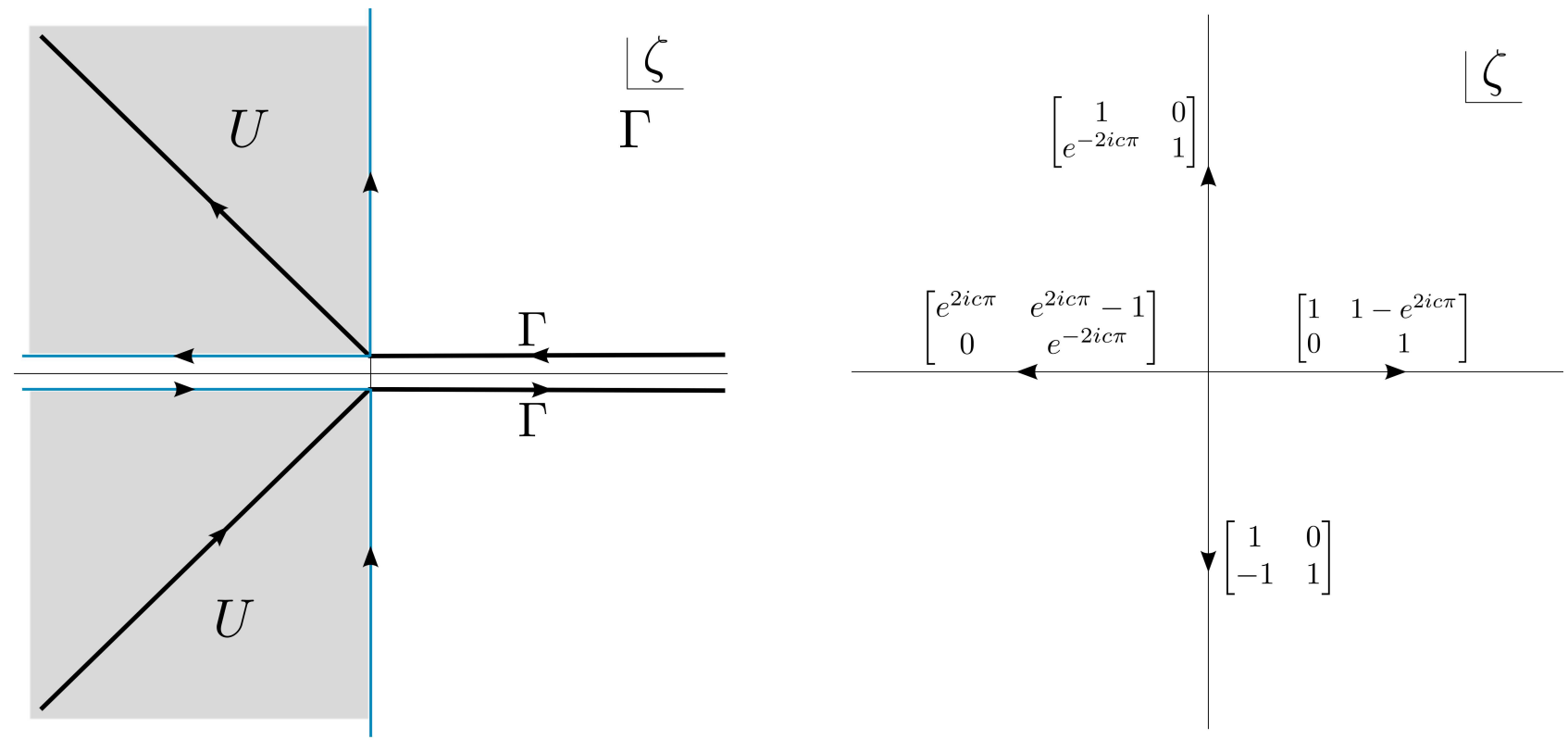

Figure 9: Jump contours of $\mathcal{P}(\underline{20})$ in $D_{\beta}$ (left) and the jump matrices of $W$ (right)

We want to transform $\mathcal{P}$ into a new matrix function, $W$, that has only constant jump matrices from the right. Such transform may be given by

$$
W(z):=\zeta(z)^{-c \sigma_{3}} S \cdot \mathcal{P}(z) \cdot T(\zeta(z))^{-1} S^{-1},
$$

using a diagonal matrix, $T$, and a piecewise constant matrix, $S$, defined below,

$$
T(\zeta)= \begin{cases}\exp \left(\frac{\zeta^{2}}{4} \sigma_{3}\right), & |\arg \zeta|<3 \pi / 4, \\ \exp \left(-\frac{\zeta^{2}}{4} \sigma_{3}\right), & \text { otherwise, }\end{cases}
$$

and

$$
S= \begin{cases}I, & \operatorname{Im} \zeta<0 \cap|\arg \zeta|<3 \pi / 4, \\
e^{c \pi \mathrm{i} \sigma_{3}}, & \operatorname{Im} \zeta>0 \cap|\arg \zeta|<3 \pi / 4, \\
{\left[\begin{array}{cc}
0 & 1 \\
-1 & 0
\end{array}\right],} & \operatorname{Im} \zeta<0 \cap|\arg \zeta| \geq 3 \pi / 4, \\
e^{c \pi \mathrm{i} \sigma_{3}}\left[\begin{array}{cc}
0 & 1 \\
-1 & 0
\end{array}\right], & \operatorname{Im} \zeta>0 \cap|\arg \zeta| \geq 3 \pi / 4 .\end{cases}
$$

Here we choose $S$ such that $S^{-1} \zeta(z)^{c \sigma_{3}}$ satisfies all the left jumps of $\mathcal{P}$, i.e.,

$$
\begin{aligned}
& \left(S^{-1} \zeta^{c \sigma_{3}}\right)_{+}=\left[\begin{array}{cc}
0 & -1 \\
1 & 0
\end{array}\right]\left(S^{-1} \zeta^{c \sigma_{3}}\right)_{-}, \quad z \in \Gamma \cap U, \\
& \left(S^{-1} \zeta^{c \sigma_{3}}\right)_{+}=e^{-c \pi \mathrm{i} \sigma_{3}}\left(S^{-1} \zeta^{c \sigma_{3}}\right)_{-}, \quad z \in \mathbb{R},
\end{aligned}
$$


such that $W$ has the jump matrices only from the right. Furthermore, the jump matrices of $W$ are constant matrices because of the right multipliction of $T^{-1}$ in (21). The jump on $\left\{t e^{ \pm \mathrm{i} 3 \pi / 4}\right\}_{0<t<\infty}$ disappears by the right multiplication by $S^{-1}$. We summarize the jump matrices of $W$ below,

$$
W_{+}(z)=W_{-}(z) \begin{cases}{\left[\begin{array}{cc}
1 & 1-e^{2 \mathrm{i} c \pi} \\
0 & 1
\end{array}\right],} & \zeta(z) \in \mathbb{R}^{+}, \\
{\left[\begin{array}{cc}
1 & 0 \\
e^{-2 \mathrm{i} c \pi} & 1
\end{array}\right],} & \zeta(z) \in \mathbb{R}^{+}, \\
{\left[\begin{array}{cc}
e^{2 \mathrm{i} c \pi} & e^{2 \mathrm{i} c \pi}-1 \\
0 & e^{-2 \mathrm{i} c \pi}
\end{array}\right],} & \zeta(z) \in \mathbb{R}^{-}, \\
{\left[\begin{array}{cc}
1 & 0 \\
-1 & 1
\end{array}\right],} & \zeta(z) \in \mathbb{R}^{-} .\end{cases}
$$

The following fact can be checked by direct calculation.

Lemma 5. For $z \in D_{\beta}$ we have

$$
\Phi(z)\left(\frac{z-a}{z}\right)^{\frac{c}{2} \sigma_{3}} S^{-1} \zeta^{c \sigma_{3}}=\left(N^{c / 2} \eta(z)\right)^{\sigma_{3}}
$$

where $\eta: D_{\beta} \rightarrow \mathbb{C}$,

$$
\eta(z):=\frac{e^{-i c \pi / 2}}{N^{c / 2}}\left(\frac{a-z}{z}\right)^{\frac{c}{2}}\left(\frac{z \zeta(z)}{z-\beta}\right)^{c}
$$

is a nonvanishing $N$-independent analytic function in $D_{\beta}$.

Using the parabolic cylinder function (9) we define $\mathcal{W}: \mathbb{C} \backslash(\mathbb{R} \cup \mathrm{i} \mathbb{R}) \rightarrow \mathbb{C}^{2 \times 2}$ as

$$
\mathcal{W}(\zeta)= \begin{cases}{\left[\begin{array}{cc}
D_{-c}(\zeta) & \frac{\mathrm{i} \sqrt{2 \pi} e^{\frac{c \pi \mathrm{i}}{2}}}{\Gamma(c)} D_{-1+c}(\mathrm{i} \zeta) \\
-\frac{\Gamma(c+1)}{\sqrt{2 \pi} e^{c \pi \mathrm{i}}} D_{-1-c}(\zeta) & e^{-\frac{c \pi \mathrm{i}}{2}} D_{c}(\mathrm{i} \zeta)
\end{array}\right],} & -\frac{\pi}{2}<\arg (\zeta)<0 \\
{\left[\begin{array}{cc}
D_{-c}(\zeta) & -\frac{\mathrm{i} \sqrt{2 \pi} e^{\frac{3 c \pi \mathrm{i}}{2}}}{\Gamma(c)} D_{-1+c}(-\mathrm{i} \zeta) \\
-\frac{\Gamma(c+1)}{\sqrt{2 \pi} e^{c \pi \mathrm{i}}} D_{-1-c}(\zeta) & e^{\frac{c \pi \mathrm{i}}{2}} D_{c}(-\mathrm{i} \zeta)
\end{array}\right],} & 0<\arg (\zeta)<\frac{\pi}{2}, \\
{\left[\begin{array}{cc}
e^{-c \pi \mathrm{i}} D_{-c}(-\zeta) & -\frac{\mathrm{i} \sqrt{2 \pi} e^{\frac{3 c \pi \mathrm{i}}{2}}}{\Gamma(c)} D_{-1+c}(-\mathrm{i} \zeta) \\
\frac{\Gamma(1+c)}{\sqrt{2 \pi} e^{2 c \pi \mathrm{i}}} D_{-1-c}(-\zeta) & e^{\frac{c \pi \mathrm{i}}{2}} D_{c}(-\mathrm{i} \zeta)
\end{array}\right],} & \frac{\pi}{2}<\arg (\zeta)<\pi, \\
{\left[\begin{array}{cc}
e^{c \pi \mathrm{i}} D_{-c}(-\zeta) & \frac{\mathrm{i} \sqrt{2 \pi} e^{\frac{c \pi \mathrm{i}}{2}}}{\Gamma(c)} D_{-1+c}(\mathrm{i} \zeta) \\
\frac{\Gamma(1+c)}{\sqrt{2 \pi}} D_{-1-c}(-\zeta) & e^{-\frac{c \pi \mathrm{i}}{2}} D_{c}(\mathrm{i} \zeta)
\end{array}\right],} & \pi<\arg (\zeta)<\frac{3 \pi}{2}\end{cases}
$$

Lemma 6. There exists the asymptotic expansion of $D_{-c}(\zeta)$ given by

$$
D_{-c}(\zeta)=e^{-\frac{\zeta^{2}}{4}} \zeta^{-c}\left(\sum_{s=0}^{n-1}(-1)^{s} \frac{(c)_{2 s}}{s !\left(2 \zeta^{2}\right)^{s}}+\varepsilon_{n}(\zeta)\right), \quad|\arg \zeta|<\frac{\pi}{2} .
$$


There exists a constant $C>0$ independent of $c$ such that

$$
\left|\varepsilon_{n}(\zeta)\right| \leq C\left|\frac{\left(\frac{c}{2}\right)_{n}\left(\frac{c+1}{2}\right)_{n}}{n !\left(\zeta^{2}\right)^{n}}\right|, \quad|\arg \zeta|<\frac{\pi}{2} .
$$

Here, $(\cdot)_{n}$ is Pochhammer's Symbol defined by $(x)_{n}=\Gamma(x+n) / \Gamma(x)$.

The proof of this lemma is in A. Though the lemma only concerns $|\arg \zeta|<\pi / 2$, this turns out to cover every term that appears in $\mathcal{W}(\zeta)$ of (25) and leads to the following lemma.

Lemma 7. $\mathcal{W}(\zeta(z))$ satisfies the jump of $W(\underline{24})$ and the asymptotic behavior

$$
\mathcal{F}(\zeta):=\mathcal{W}(\zeta) \zeta^{c \sigma_{3}} e^{\frac{\zeta^{2}}{4} \sigma_{3}}=I+\frac{C_{1}}{\zeta}+\frac{C_{2}}{\zeta^{2}}+\mathcal{O}\left(\frac{1}{\zeta^{3}}\right)
$$

as $|\zeta|$ goes to $\infty$, where

$$
C_{1}=\left[\begin{array}{cc}
0 & \frac{\sqrt{2 \pi} e^{\mathrm{i} \pi c}}{\Gamma(c)} \\
-\frac{\Gamma(c+1)}{\sqrt{2 \pi} e^{\mathrm{i} \pi c}} & 0
\end{array}\right] \quad \text { and } \quad C_{2}=\left[\begin{array}{cc}
-\frac{c(c+1)}{2} & 0 \\
0 & \frac{c(c-1)}{2}
\end{array}\right]
$$

Moreover, as $c \rightarrow 0$ and $|\zeta| \rightarrow \infty$, we get

$$
\begin{aligned}
& \mathcal{F}(\zeta) F_{1}(\zeta)^{-1}=I+\left[\begin{array}{ll}
\mathcal{O}\left(c \zeta^{-2}\right) & \mathcal{O}\left(c \zeta^{-3}\right) \\
\mathcal{O}\left(\zeta^{-1}\right) & \mathcal{O}\left(c \zeta^{-2}\right)
\end{array}\right], \\
& \mathcal{F}(\zeta) F_{1}(\zeta)^{-1} F_{2}(\zeta)^{-1}=I+\mathcal{O}\left(\zeta^{-3}\right),
\end{aligned}
$$

where

$$
\begin{aligned}
& F_{1}(\zeta)=I+\frac{1}{\zeta}\left[\begin{array}{cc}
0 & \frac{\sqrt{2 \pi} e^{\mathrm{i} \pi c}}{\Gamma(c)} \\
0 & 0
\end{array}\right], \\
& F_{2}(\zeta)=I+\left[\begin{array}{cc}
-\frac{c(c+1)}{2} \frac{1}{\zeta^{2}} & \frac{1}{\zeta^{3}} \frac{\sqrt{2 \pi} e^{\mathrm{i} \pi c} c^{2}(c+1)^{2}}{4 \Gamma(c+1)} \\
-\frac{\Gamma(c+1)}{\sqrt{2 \pi} e^{\mathrm{i} \pi c} \frac{1}{\zeta}} & \frac{c(c+1)}{2} \frac{1}{\zeta^{2}}
\end{array}\right] .
\end{aligned}
$$

The error bound in (28) is uniform over $c \in[-1 / 2,1 / 2]$ as $\zeta$ tends to infinity, and the error bound in (29) is for a fixed $c$.

Proof. The proof of the jump is an exercise using the following identities [20, 6]:

$$
\begin{aligned}
& D_{-c}(\zeta)=\frac{\Gamma(1-c)}{\sqrt{2 \pi}}\left[e^{\frac{-c \pi \mathrm{i}}{2}} D_{c-1}(\mathrm{i} \zeta)+e^{\frac{c \pi \mathrm{i}}{2}} D_{c-1}(-\mathrm{i} \zeta)\right] \\
& D_{-c}(\zeta)=e^{-c \pi \mathrm{i}} D_{-c}(-\zeta)+\frac{\sqrt{2 \pi}}{\Gamma(c)} e^{\frac{(1-c) \pi \mathrm{i}}{2}} D_{c-1}(-\mathrm{i} \zeta) \\
& D_{-c}(\zeta)=e^{c \pi \mathrm{i}} D_{-c}(-\zeta)+\frac{\sqrt{2 \pi}}{\Gamma(c)} e^{\frac{(c-1) \pi \mathrm{i}}{2}} D_{c-1}(\mathrm{i} \zeta)
\end{aligned}
$$


The proof of the asymptotic behavior is based on Lemma 6 about the asymptotic behavior of the parabolic cylinder function. By Lemma 6 , letting $n=1$, we have

$$
\left|\varepsilon_{1}(\zeta)\right| \leq C\left|\frac{c(c+1)}{\zeta^{2}}\right|, \quad|\arg \zeta|<\frac{\pi}{2} .
$$

This leads to $D_{-c}(\zeta)=e^{-\zeta^{2} / 4} \zeta^{-c}\left(1+\mathcal{O}\left(c(c+1) / \zeta^{2}\right)\right)$. Similarly, we can obtain the asymptotic expression for $D_{-1+c}(\mathrm{i} \zeta), D_{-1-c}(\zeta)$, and $D_{c}(\mathrm{i} \zeta)$ and we get

$$
\mathcal{F}(\zeta)=F_{1}(\zeta)+\left[\begin{array}{cc}
\mathcal{O}\left(\frac{c(c+1)}{\zeta^{2}}\right) & \mathcal{O}\left(\frac{(c-1)(c-2)}{\zeta^{3} \Gamma(c)}\right) \\
\mathcal{O}\left(\frac{\Gamma(c+1)}{\zeta}\right) & \mathcal{O}\left(\frac{c(c-1)}{\zeta^{2}}\right)
\end{array}\right]
$$

This leads to (28) using $\Gamma(c)=c^{-1}(1+\mathcal{O}(c))$. Similarly, the equations (29) and (27) follow from Lemma 6 .

Let $H$ be a unimodular holomorphic matrix function on $D_{\beta}$. We define $W$ by

$$
W(z)=H(z) \mathcal{W}(\zeta(z)), \quad z \in D_{\beta} .
$$

Combining (21), (27) and (32), the expression in (19) can be written as

$$
\begin{aligned}
& \Phi(z)\left(\frac{z-a}{z}\right)^{\frac{c}{2} \sigma_{3}} \mathcal{P}(z)\left(\frac{z-a}{z}\right)^{-\frac{c}{2} \sigma_{3}} \\
& =\Phi(z)\left(\frac{z-a}{z}\right)^{\frac{c}{2} \sigma_{3}} S^{-1} \zeta^{c \sigma_{3}} H(z) \mathcal{W}(z) S T(\zeta(z))\left(\frac{z-a}{z}\right)^{-\frac{c}{2} \sigma_{3}} \\
& =\Phi(z)\left(\frac{z-a}{z}\right)^{\frac{c}{2} \sigma_{3}} S^{-1} \zeta^{c \sigma_{3}} H(z) \mathcal{F}(\zeta(z)) \zeta(z)^{-c \sigma_{3}} e^{-\frac{\zeta(z)^{2}}{4} \sigma_{3}} S T(\zeta(z))\left(\frac{z-a}{z}\right)^{-\frac{c}{2} \sigma_{3}} .
\end{aligned}
$$

By (22), (23) and Lemma 5, we obtain

$$
\zeta^{-c \sigma_{3}} e^{\frac{-\zeta^{2}}{4} \sigma_{3}} S T(\zeta(z))\left(\frac{z-a}{z}\right)^{-\frac{c}{2} \sigma_{3}}=\zeta^{-c \sigma_{3}} S\left(\frac{z-a}{z}\right)^{-\frac{c}{2} \sigma_{3}}=\left(N^{c / 2} \eta(z)\right)^{-\sigma_{3}} \Phi(z) .
$$

The above equations lead to the following Lemma.

Lemma 8. When $z \in D_{\beta}$, we have

$$
\Phi(z)\left(\frac{z-a}{z}\right)^{\frac{c}{2} \sigma_{3}} \mathcal{P}(z)\left(\frac{z-a}{z}\right)^{-\frac{c}{2} \sigma_{3}}=\left(N^{c / 2} \eta(z)\right)^{\sigma_{3}} H(z) \mathcal{F}(\zeta(z))\left(N^{c / 2} \eta(z)\right)^{-\sigma_{3}} \Phi(z) .
$$


Theorem 4. For $a>1$ and $-1 / 2 \leq c \leq 1 / 2$, we get

$$
P_{N}(z)=\left\{\begin{array}{rlrl}
z^{N}\left(\frac{z}{z-\beta}\right)^{c}\left(1+\mathcal{O}\left(\frac{1}{N^{c+1 / 2}}\right)\right), & z \in \operatorname{Ext} \mathcal{S} \backslash\left(U \cup D_{\beta}\right), \\
z^{N}\left(\left(\frac{z}{z-\beta}\right)^{c}-\frac{\sqrt{2 \pi}\left(a^{2}-1\right)^{c}}{N^{1 / 2-c} a \Gamma(c)} \frac{e^{N \phi_{A}(z)}}{(z-\beta)}\left(\frac{z-\beta}{z-a}\right)^{c}\right. & z \in U \backslash D_{\beta}, \\
& \left.+\mathcal{O}\left(\frac{1}{N^{c+1 / 2}}, \frac{e^{N \phi_{A}}}{N^{c+1 / 2}}\right)\right), & \\
z^{N}\left(\left(\frac{z \zeta}{z-\beta}\right)^{c} e^{\frac{\zeta^{2}(z)}{4}} D_{-c}(\zeta(z))+\mathcal{O}\left(\frac{1}{N^{1 / 2}}, \frac{1}{N^{2 c+1 / 2}}\right)\right), & z \in D_{\beta} .
\end{array}\right.
$$

The error bounds are uniform in $c \in[-1 / 2,1 / 2]$. The big $\mathcal{O}$ notation with multiple arguments is defined by $\mathcal{O}(A, B)=\mathcal{O}(A)+\mathcal{O}(B)$.

This theorem is similar to Theorem 2 except that the range of $c$ is restricted to $[-1 / 2,1 / 2]$ and the error bounds are uniform in the range.

Proof. Using $F_{1}$ in (30) we can define a unimodular meromorphic matrix function with a simple pole at $\beta$ by

$$
\mathcal{R}(z)=I+\frac{\sqrt{2 \pi}\left(a^{2}-1\right)^{c}}{N^{1 / 2-c} a \Gamma(c)} \frac{1}{z-\beta}\left[\begin{array}{ll}
0 & 1 \\
0 & 0
\end{array}\right]
$$

such that we can set

$$
H(z)=\left(N^{c / 2} \eta(z)\right)^{-\sigma_{3}} \mathcal{R}(z)\left(N^{c / 2} \eta(z)\right)^{\sigma_{3}} F_{1}(\zeta(z))^{-1}
$$

i.e., the above is unimodular and holomorphic at $\beta$.

Now we define the strong asymptotics of $Z$ that we will denote by

$$
Z^{\infty}(z):= \begin{cases}\mathcal{R}(z) \Phi(z), & z \notin D_{\beta}, \\ \Phi(z)\left(\frac{z-a}{z}\right)^{\frac{c}{2} \sigma_{3}} \mathcal{P}(z)\left(\frac{z-a}{z}\right)^{-\frac{c}{2} \sigma_{3}}, & z \in D_{\beta},\end{cases}
$$

where the second line is given in Lemma 8 , We get

$$
\begin{aligned}
Z_{+}^{\infty}(z)\left(Z_{-}^{\infty}(z)\right)^{-1} & =\Phi(z)\left(\frac{z-a}{z}\right)^{\frac{c}{2} \sigma_{3}} \mathcal{P}(z)\left(\frac{z-a}{z}\right)^{-\frac{c}{2} \sigma_{3}} \Phi^{-1}(z) \mathcal{R}^{-1}(z) \\
& =\left(N^{c / 2} \eta(z)\right)^{\sigma_{3}} H(z) \mathcal{F}(z)\left(N^{c / 2} \eta(z)\right)^{-\sigma_{3}} \mathcal{R}^{-1}(z) \\
& =\left(N^{c / 2} \eta(z)\right)^{\sigma_{3}} H(z) \widehat{\mathcal{F}}(\zeta) H^{-1}(z)\left(N^{c / 2} \eta(z)\right)^{-\sigma_{3}}
\end{aligned}
$$

where, in the last line, we define

$$
\widehat{\mathcal{F}}(\zeta)=\mathcal{F}(\zeta) F_{1}(\zeta)^{-1}
$$


Defining the error matrix by

$$
\mathcal{E}(z):=Z^{\infty}(z) Z^{-1}(z)
$$

we get

$$
\begin{aligned}
\mathcal{E}_{+}(z) \mathcal{E}_{-}^{-1}(z) & =Z^{\infty}(z)_{+}\left(Z_{-}^{\infty}(z)\right)^{-1} \\
& =\left(N^{c / 2} \eta(z)\right)^{\sigma_{3}} H(z) \widehat{\mathcal{F}}(\zeta) H^{-1}(z)\left(N^{c / 2} \eta(z)\right)^{-\sigma_{3}} \\
& =I+\left[\begin{array}{cc}
\mathcal{O}\left(\frac{c}{N}\right) & \mathcal{O}\left(\frac{c}{N^{3 / 2-c}}\right) \\
\mathcal{O}\left(\frac{1}{N^{1 / 2+c}}\right) & \mathcal{O}\left(\frac{c}{N}\right)
\end{array}\right]=I+\mathcal{O}\left(\frac{1}{N^{1 / 2+c}}\right), \quad z \in \partial D_{\beta},
\end{aligned}
$$

where, in the last equality, we use the asymptotic behavior (28) for $\widehat{\mathcal{F}}(\zeta)=\mathcal{F}(\zeta) F_{1}(\zeta)^{-1}$, and the asymptotic behavior of $H$ given below.

$$
H=\left[\begin{array}{cc}
1 & h(z) \\
0 & 1
\end{array}\right], \quad h(z)=\frac{\sqrt{2 \pi}\left(a^{2}-1\right)^{c}}{\sqrt{N} \eta^{2}(z) a \Gamma(c)} \frac{1}{z-\beta}-\frac{1}{\zeta(z)} \frac{\sqrt{2 \pi} e^{\mathrm{i} \pi c}}{\Gamma(c)}=\mathcal{O}\left(\frac{c}{\sqrt{N}}\right) .
$$

One can check that the jump of $\mathcal{E}$ is exponentially small in $N$ away from $\partial D_{\beta}$ using Lemma 4 and (18). By the small norm theorem (e.g. Theorem 7.171 in [7] or [8]) we obtain $\mathcal{E}(z)=I+\mathcal{O}\left(1 / N^{c+1 / 2}\right)$ and, therefore, $Z^{\infty}(z) Z^{-1}(z)=I+\mathcal{O}\left(1 / N^{c+1 / 2}\right)$. Note that the error bound is uniform over $c \in[-1 / 2,1 / 2]$.

Using (17) we have (see (17) for the definition of $\star$ )

$$
\begin{aligned}
& Y(z)=e^{\frac{N \ell}{2} \sigma_{3}} Z(z)\left[-\star\left(\frac{z}{z-a}\right)^{c} e^{N \phi(z)} \quad 1\right] e^{\frac{-N \ell}{2} \sigma_{3}} e^{N g(z) \sigma_{3}} \\
& =e^{\frac{N \ell}{2} \sigma_{3}}\left(I+\mathcal{O}\left(\frac{1}{N^{1 / 2+c}}\right)\right) Z^{\infty}(z)\left[-\star\left(\frac{z}{z-a}\right)^{c} e^{N \phi(z)} \quad 1\right] e^{\frac{-N \ell}{2} \sigma_{3}} e^{N g(z) \sigma_{3}} .
\end{aligned}
$$

Using (36), we calculate the strong asymptotics for $z \in(\operatorname{Ext} \mathcal{S} \cap U) \backslash D_{\beta}$ as an example.

$$
\begin{aligned}
& P_{N}(z)=[Y(z)]_{11}=\left[\left(I+\mathcal{O}\left(\frac{1}{N^{1 / 2+c}}\right)\right) Z^{\infty}(z)\left[\begin{array}{cc}
1 & 0 \\
-\star\left(\frac{z}{z-a}\right)^{c} e^{N \phi(z)} & 1
\end{array}\right]\right]_{11} e^{N g(z)} \\
& =\left[\left(I+\mathcal{O}\left(\frac{1}{N^{1 / 2+c}}\right)\right) \mathcal{R}(z) \Phi(z)\left[-\left(\frac{z}{z-a}\right)^{c} e^{N \phi(z)} \quad 1\right]\right]_{11} e^{N g(z)} \\
& =\left[\left(I+\mathcal{O}\left(\frac{1}{N^{1 / 2+c}}\right)\right)\left[\begin{array}{cc}
1 & \frac{\sqrt{2 \pi}\left(a^{2}-1\right)^{c}}{N^{1 / 2-c} a \Gamma(c)} \frac{1}{z-\beta} \\
0 & 1
\end{array}\right]\left[\begin{array}{cc}
\left(\frac{z}{z-\beta}\right)^{c} & 0 \\
0 & \left(\frac{z-\beta}{z}\right)^{c}
\end{array}\right]\left[\begin{array}{cc}
1 & 0 \\
-\left(\frac{z}{z-a}\right)^{c} e^{N \phi(z)} & 1
\end{array}\right]\right]_{11} z^{N} \\
& =\left[\left(1+\mathcal{O}\left(\frac{1}{N^{1 / 2+c}}\right)\right)\left(\left(\frac{z}{z-\beta}\right)^{c}-\left(\frac{z-\beta}{z-a}\right)^{c} \frac{\sqrt{2 \pi}\left(a^{2}-1\right)^{c}}{a \Gamma(c) N^{1 / 2-c}(z-\beta)} e^{N \phi(z)}\right)\right. \\
& \left.-\mathcal{O}\left(\frac{1}{N^{1 / 2+c}}\right)\left(\frac{z-\beta}{z-a}\right)^{c} e^{N \phi(z)}\right] z^{N} \\
& =z^{N}\left(\left(\frac{z}{z-\beta}\right)^{c}-\left(\frac{z-\beta}{z-a}\right)^{c} \frac{\sqrt{2 \pi}\left(a^{2}-1\right)^{c}}{a \Gamma(c) N^{1 / 2-c}(z-\beta)} e^{N \phi(z)}+\mathcal{O}\left(\frac{1}{N^{1 / 2+c}}\right)\right) .
\end{aligned}
$$


A similar calculation will give the following for $z \in(\operatorname{Int} \mathcal{S} \cap U) \backslash D_{\beta}$ :

$$
P_{N}(z)=e^{N g(z)}\left(\left(\frac{z}{z-\beta}\right)^{c} e^{N \phi(z)}-\left(\frac{z-\beta}{z-a}\right)^{c} \frac{\sqrt{2 \pi}\left(a^{2}-1\right)^{c}}{a \Gamma(c) N^{1 / 2-c}(z-\beta)}+\mathcal{O}\left(\frac{1}{N^{1 / 2+c}}\right)\right) .
$$

For $z \in(\operatorname{Ext} \mathcal{S} \backslash U) \cap D_{\beta}$ we calculate the strong asymptotics using (36), (27) and Lemma 8 to represent $\mathcal{P}$ in terms of $\mathcal{W}(25)$ and $H(z)(35)$.

$$
\begin{aligned}
& P_{N}(z)=[Y(z)]_{11}=\left[\left(I+\mathcal{O}\left(\frac{1}{N^{1 / 2+c}}\right)\right) Z^{\infty}\left[\begin{array}{cc}
1 & 0 \\
-\star\left(\frac{z}{z-a}\right)^{c} e^{N \phi(z)} & 1
\end{array}\right]\right]_{11} e^{N g(z)} \\
& =\left[\left(I+\mathcal{O}\left(\frac{1}{N^{1 / 2+c}}\right)\right) \Phi(z)\left(\frac{z-a}{z}\right)^{\frac{c}{2} \sigma_{3}} \mathcal{P}(z)\left(\frac{z-a}{z}\right)^{-\frac{c}{2} \sigma_{3}}\right]_{11} z^{N} \\
& =\left[\left(I+\mathcal{O}\left(\frac{1}{N^{1 / 2+c}}\right)\right)\left(N^{c / 2} \eta(z)\right)^{\sigma_{3}} H(z) \mathcal{F}(\zeta(z))\left(N^{c / 2} \eta(z)\right)^{-\sigma_{3}} \Phi(z)\right]_{11} z^{N} \\
& =\left[\left(I+\mathcal{O}\left(\frac{1}{N^{1 / 2+c}}\right)\right)\left(N^{c / 2} \eta(z)\right)^{\sigma_{3}}\left[\begin{array}{cc}
1 & h(z) \\
0 & 1
\end{array}\right]\left[\begin{array}{cc}
D_{-c}(\zeta) & \frac{\mathrm{i} \sqrt{2 \pi} e^{\frac{c \pi \mathrm{i}}{2}}}{\Gamma(c)} D_{-1+c}(\mathrm{i} \zeta) \\
-\frac{\Gamma(c+1)}{\sqrt{2 \pi} e^{c \pi \mathrm{i}}} D_{-1-c}(\zeta) & e^{-\frac{c \pi \mathrm{i}}{2}} D_{c}(\mathrm{i} \zeta)
\end{array}\right]\right. \\
& \left.\cdot \zeta^{c \sigma_{3}} e^{\frac{\zeta^{2}}{4} \sigma_{3}}\left(N^{c / 2} \eta(z)\right)^{-\sigma_{3}}\left[\begin{array}{cc}
\left(\frac{z}{z-\beta}\right)^{c} & 0 \\
0 & \left(\frac{z-\beta}{z}\right)^{c}
\end{array}\right]\right]_{11} z^{N} \\
& =\left[\left(\frac{z}{z-\beta}\right)^{c} \zeta(z)^{c} e^{\frac{\zeta(z)^{2}}{4}}\left(D_{-c}(\zeta)-h(z) \frac{\Gamma(c+1)}{\sqrt{2 \pi} e^{c \pi \mathrm{i}}} D_{-1-c}(\zeta)\right)\left(1+\mathcal{O}\left(\frac{1}{N^{1 / 2+c}}\right)\right)\right. \\
& \left.+\mathcal{O}\left(\frac{1}{N^{1 / 2+2 c}}\right)\right] z^{N} \\
& =\left[\left(\frac{z}{z-\beta}\right)^{c} \zeta(z)^{c} e^{\frac{\zeta(z)^{2}}{4}} D_{-c}(\zeta)+\mathcal{O}\left(\frac{1}{\sqrt{N}}, \frac{1}{N^{1 / 2+2 c}}\right)\right] z^{N} .
\end{aligned}
$$

We used (39) at the last equality. Note that the above error bounds are uniform over $c \in[-1 / 2,1 / 2]$.

For the other regions we skip the calculations as they are similar.

\section{$5 \quad a>1$ : Proof of Theorem 2}

The proof of Theorem 2 is identical to the above proof of Theorem 4 except that we use different $\mathcal{R}$ and $H$ (hence different $\mathcal{P}$ ). The construction of $\mathcal{R}$ and $H$ will be more involved and will be useful for the next case of $a<1$ and, therefore, we will describe the construction in a more general setting.

Here we describe how to construct $\mathcal{R}$ and $\mathcal{P}$ inductively such that the jump, $Z_{+}^{\infty}\left(Z_{-}^{\infty}\right)^{-1}$, of $Z^{\infty}$ is close to the identity up to $\mathcal{O}\left(N^{-L}\right)$ for any given $L>0$. The inductive method that we describe here involves only algebraic manipulations - such as the inverse of relatively small matrices.

We introduce several notations that we will use in this section.

Let us recall that $\zeta$ is a univalent function in $D_{\beta}$ such that $\zeta(\beta)=0$ and $N^{-\tau_{a}} \zeta(z) /(z-\beta)$ is an $N$-independent and non-vanishing holomorphic function where (we include the case, $a<1$, for later)

$$
\tau_{a}= \begin{cases}1 / 2 & \text { for } a>1 \\ 1 & \text { for } a<1\end{cases}
$$


The lemma below generalize the definition of $\widehat{\mathcal{F}}$ that we used in the previous section.

Lemma 9. Let $\mathcal{F}$ be a unimodular piecewise analytic matrix function with the asymptotic expansion around $\infty$ given by

$$
\mathcal{F}=I+\frac{C_{1}}{\zeta}+\frac{C_{2}}{\zeta^{2}}+\cdots
$$

where $C_{j}$ 's are constant $2 \times 2$ matrices. For any positive integer $L$, there exists a positive number of $k$ and a decomposition

$$
\mathcal{F}(\zeta)=\widehat{\mathcal{F}}(\zeta) F_{k}(\zeta) \cdots F_{1}(\zeta)
$$

such that, for all $1 \leq j \leq k, F_{j}$ is a rational function with only singularity at the origin, $F_{j}(\infty)=I$, $F_{j}(\zeta)-I$ is nilpotent and

$$
\widehat{\mathcal{F}}(\zeta)=I+\mathcal{O}\left(\zeta^{-L}\right)
$$

Proof. Assume

$$
\mathcal{F}(\zeta)=I+\frac{C_{0}}{\zeta^{m}}+\mathcal{O}\left(\frac{1}{\zeta^{m+1}}\right), \quad C_{0}=\left[\begin{array}{ll}
c_{11} & c_{12} \\
c_{21} & c_{22}
\end{array}\right] .
$$

Since $\operatorname{det} \mathcal{F}=1$, we have $c_{11}+c_{22}=0$. One can write $C_{0}$ as the sum of three nilpotent matrices, $C_{0}=N_{1}+N_{2}+N_{3}$, where

$$
N_{1}=\left[\begin{array}{cc}
c_{11} & -c_{11}^{2} \\
1 & -c_{11}
\end{array}\right], \quad N_{2}=\left[\begin{array}{cc}
0 & c_{12}-c_{11}^{2} \\
0 & 0
\end{array}\right], \quad N_{3}=\left[\begin{array}{cc}
0 & 0 \\
c_{21}-1 & 0
\end{array}\right] .
$$

We get

$$
\mathcal{F}(\zeta)\left(I+\frac{N_{1}}{\zeta^{m}}\right)^{-1}\left(I+\frac{N_{2}}{\zeta^{m}}\right)^{-1}\left(I+\frac{N_{3}}{\zeta^{m}}\right)^{-1}=I+\mathcal{O}\left(\frac{1}{\zeta^{m+1}}\right)
$$

Using induction, this proves the lemma.

Given $\left\{F_{k}\right\}_{k=1,2, \cdots}$, we will define $\left\{H_{k}\right\}$ and $\left\{R_{k}\right\}$ inductively. Let $H_{0}=I$. Assume that $H_{k-1}$ is holomorphic and non-vanishing at $\beta$, and $H_{k-1}(z)=I+\mathcal{O}\left(1 / N^{\tau_{a}}\right)$. We define

$$
\widetilde{F}_{k}(z):=\left(N^{\frac{c}{2}} \eta(z)\right)^{\sigma_{3}} H_{k-1}(z) F_{k}(\zeta(z)) H_{k-1}^{-1}(z)\left(N^{\frac{c}{2}} \eta(z)\right)^{-\sigma_{3}} .
$$

If $F_{k}$ satisfies the property described in Lemma 9, we have the following truncated Laurent series expansion near $\beta$,

$$
\widetilde{F}_{k}^{-1}(z)=N^{\frac{c}{2} \sigma_{3}}\left(I+\sum_{j=-\infty}^{m_{k}} \frac{A_{j}}{(z-\beta)^{j}}\right) N^{-\frac{c}{2} \sigma_{3}},
$$

for some positive integer $m_{k}$ and some constant matrices $\left\{A_{j}\right\}$. Given $\left\{A_{j}\right\}$, the lemma below constructs $\left\{R_{k}\right\}$ inductively.

Lemma 10. Given $\widetilde{F}_{k}(z)$ as above, the unique rational matrix function $R_{k}$ such that its only singularity is at $\beta, R_{k}(\infty)=I$ and $R_{k}(z) \widetilde{F}_{k}^{-1}(z)$ is holomorphic at $\beta$, is given by

$$
R_{k}(z)=N^{\frac{c}{2} \sigma_{3}}\left(I+\sum_{j=1}^{m_{k}} \frac{B_{j}}{(z-\beta)^{j}}\right) N^{-\frac{c}{2} \sigma_{3}},
$$


where, for a sufficiently large $N, B_{j}$ 's are given by

$$
\left[B_{m_{k}}, B_{m_{k-1}}, \cdots, B_{1}\right]=-\left[A_{m_{k}}, A_{m_{k-1}}, \cdots, A_{1}\right](I+\widetilde{M})^{-1} .
$$

The $2 m_{k} \times 2 m_{k}$ matrix $\widetilde{M}$ is given in block form by

$$
\widetilde{M}=\left[\begin{array}{cccc}
A_{0} & A_{-1} & \cdots & A_{1-m_{k}} \\
A_{1} & A_{0} & \cdots & A_{2-m_{k}} \\
\vdots & \ddots & \ddots & \vdots \\
A_{m_{k-1}} & \cdots & A_{1} & A_{0}
\end{array}\right]
$$

and, for a sufficiently large $N, I+\widetilde{M}$ is invertible. Moreover, $\operatorname{det} R_{k} \equiv 1$.

Proof. Let

$$
M=\left[\begin{array}{cccc}
A_{m_{k}} & A_{m_{k-1}} & \cdots & A_{1} \\
& A_{m_{k}} & \cdots & A_{2} \\
& & \ddots & \vdots \\
& & & A_{m_{k}}
\end{array}\right],
$$

In order to make $R_{k}(z) \widetilde{F}_{k}^{-1}(z)$ holomorphic at $\beta$, we require all the pole terms of $R_{k}(z) \widetilde{F}_{k}^{-1}(z)$ to vanish. We obtain

$$
\begin{aligned}
& {\left[B_{m_{k}}, B_{m_{k-1}}, \cdots, B_{1}\right] \cdot M=0,} \\
& {\left[B_{m_{k}}, B_{m_{k-1}}, \cdots, B_{1}\right](I+\widetilde{M})+\left[A_{m_{k}}, A_{m_{k-1}}, \cdots, A_{1}\right]=0,}
\end{aligned}
$$

where the first equation comes from the the poles of the orders $2 m_{k}, 2 m_{k}-1, \cdots, m_{k}+1$, and the second equation comes from the poles orders $m_{k}, m_{k}-1, \cdots, 1$.

We explain a useful bound on $A_{j}$ 's. If $F_{k}(\zeta)=I+\mathcal{O}\left(\zeta^{-m_{k}}\right)$, then $F_{k}(\zeta(z))=I+\mathcal{O}\left(N^{-m_{k} \tau_{a}}\right)$ on $\partial D_{\beta}$. Therefore, we have $A_{j}=\mathcal{O}\left(N^{-m_{k} \tau_{a}}\right)$ and $\|\widetilde{M}\|=\mathcal{O}\left(N^{-m_{k} \tau_{a}}\right)$. Hence $I+\widetilde{M}$ is invertible for a sufficiently large $N$ so that, from (45), we can obtain

$$
\left[B_{m_{k}}, B_{m_{k-1}}, \cdots, B_{1}\right]=-\left[A_{m_{k}}, A_{m_{k-1}}, \cdots, A_{1}\right](I+\widetilde{M})^{-1} .
$$

Let us show that (44) is satisfied. Since $F_{k}(\zeta)-I$ is nilpotent, $\widetilde{F}_{k}^{-1}(z)-I$ is nilpotent and, therefore,

$$
\left(\sum_{j=-\infty}^{m_{k}} \frac{A_{j}}{(z-\beta)^{j}}\right)^{2}=0 .
$$


This implies $M^{2}=0$ and $M \widetilde{M}=-\widetilde{M} M$. Then,

$$
\begin{aligned}
{\left[B_{m_{k}}, B_{m_{k-1}}, \cdots, B_{1}\right] \cdot M } & =-\left[A_{m_{k}}, A_{m_{k-1}}, \cdots, A_{1}\right](I+\widetilde{M})^{-1} \cdot M \\
& =-[M]_{1 \text { st row }}\left(I-\widetilde{M}+\widetilde{M}^{2}+\cdots\right) \cdot M \\
& =-\left[M \cdot\left(I-\widetilde{M}+\widetilde{M}^{2}+\cdots\right) \cdot M\right]_{1 \text { st row }} \\
& =-\left[M M-M \widetilde{M} M+M \widetilde{M}^{2} M+\cdots\right]_{1 \text { st row }} \\
& =-\left[M M+M^{2} \widetilde{M}+M^{2} \widetilde{M}^{2}+\cdots\right]_{1 \text { st row }}=0
\end{aligned}
$$

The "1st row" means the 1 st two rows or, equivalently, the 1 st row in the $2 \times 2$ block matrix. Since $R_{k}(z) \widetilde{F}_{k}^{-1}(z)$ is holomorphic at $\beta$ and $\operatorname{det} \widetilde{F}_{k}^{-1}(z) \equiv 1$, $\operatorname{det} R_{k}(z)$ is holomorphic at $\beta$. Since $\operatorname{det} R_{k}(\infty)=1$, we have $\operatorname{det} R_{k} \equiv 1$.

Now we show that $R_{k}$ is unique. Assume $\widetilde{R}_{k}$ also satisfies all the conditions satisfied by $R_{k}$ in the lemma. Then, $R_{k} \widetilde{R}_{k}^{-1}$ is holomorphic away from $\beta, R_{k}(z) \widetilde{R}_{k}(z)^{-1} \rightarrow I$ as $z \rightarrow \infty$, and $R_{k} \widetilde{R}_{k}^{-1}=$ $R_{k} \widetilde{F}_{k}^{-1}\left(\widetilde{R}_{k} \widetilde{F}_{k}^{-1}\right)^{-1}$ is holomorphic at $\beta$. Thus, $R_{k}=\widetilde{R}_{k}$.

Corollary 1. If $F_{k}(\zeta)=I+\mathcal{O}\left(\zeta^{-m}\right)$, then $N^{-\frac{c}{2} \sigma_{3}} R_{k}(z) N^{\frac{c}{2} \sigma_{3}}=I+\mathcal{O}\left(N^{-\tau_{a} m}\right)$ when $z \in \partial D_{\beta}$.

Proof. From $A_{j}=\mathcal{O}\left(N^{-m \tau_{a}}\right), B_{j}=\mathcal{O}\left(N^{-m \tau_{a}}\right)$ follows.

Using $R_{k}(z)$ from the above lemma, we define $H_{k}(z)$ by

$$
H_{k}(z)=\left(N^{\frac{c}{2}} \eta(z)\right)^{-\sigma_{3}} R_{k}(z) \widetilde{F}_{k}^{-1}(z)\left(N^{\frac{c}{2}} \eta(z)\right)^{\sigma_{3}} H_{k-1}(z) .
$$

Since $H_{0}=I$, by induction, $H_{k}(z)$ is holomorphic at $\beta$ and unimodular. By Corollary 1 we get

$$
H_{k}(z)=I+\mathcal{O}\left(N^{-\tau_{a}}\right), \quad z \in \overline{D_{\beta}} .
$$

Lemma 11. For $z \in \partial D_{\beta}$, we have

$$
Z_{+}^{\infty}(z)\left(Z_{-}^{\infty}(z)\right)^{-1}=\left(N^{c / 2} \eta(z)\right)^{\sigma_{3}} H(z) \widehat{\mathcal{F}}(\zeta) H^{-1}(z)\left(N^{c / 2} \eta(z)\right)^{-\sigma_{3}} .
$$

Proof. We have

$$
\begin{aligned}
Z_{+}^{\infty}(z)\left(Z_{-}^{\infty}(z)\right)^{-1} & =\Phi(z)\left(\frac{z-a}{z}\right)^{\frac{c}{2} \sigma_{3}} \mathcal{P}(z)\left(\frac{z-a}{z}\right)^{-\frac{c}{2} \sigma_{3}} \Phi^{-1}(z) \mathcal{R}^{-1}(z) \\
& =\left(N^{c / 2} \eta(z)\right)^{\sigma_{3}} H(z) \mathcal{F}(z)\left(N^{c / 2} \eta(z)\right)^{-\sigma_{3}} \mathcal{R}^{-1}(z) \\
& =\left(N^{c / 2} \eta(z)\right)^{\sigma_{3}} H(z) \widehat{\mathcal{F}}(\zeta) H^{-1}(z)\left(N^{c / 2} \eta(z)\right)^{-\sigma_{3}}
\end{aligned}
$$

The first equality is from (36), the second equality comes from Lemma 8 , and the last equality follows from (42) and

$$
H=H_{k}=\left(N^{c / 2} \eta\right)^{-\sigma_{3}} R_{k} \cdots R_{1}\left(N^{c / 2} \eta\right)^{\sigma_{3}} F_{1}^{-1} \cdots F_{k}^{-1}
$$

which follows from the inductive definition of $H_{k}$ at (46) with $H_{0}=I$. The theorem is proved using Lemma 9 and (47). 
Proof of Theorem 2. Contrary to the proof of Theorem 4, all the error bounds will be for a fixed $c$.

Here, we construct $\left\{R_{j}\right\}$ and $\left\{H_{j}\right\}$ inductively from the initial data $R_{1}=\mathcal{R}$ and $H_{1}=H$ where $\mathcal{R}$ and $H$ given in (34) and (35).

By (31) with (43) a calculation leads to,

$$
\begin{aligned}
\widetilde{F}_{2}(z) & =\left(N^{\frac{c}{2}} \eta(z)\right)^{\sigma_{3}} H_{1}(z) F_{2}(\zeta(z)) H_{1}^{-1}(z)\left(N^{\frac{c}{2}} \eta(z)\right)^{-\sigma_{3}} \\
& =N^{\frac{c}{2} \sigma_{3}}\left(I+\left[\begin{array}{cc}
\mathcal{O}\left(\frac{1}{N}\right) & \mathcal{O}\left(\frac{1}{N^{3 / 2}}\right) \\
\mathcal{O}\left(\frac{1}{\sqrt{N}}\right) & \mathcal{O}\left(\frac{1}{N}\right)
\end{array}\right]\right) N^{-\frac{c}{2} \sigma_{3}} . \quad z \in \partial D_{\beta} .
\end{aligned}
$$

An estimate using $H_{1}=I+\mathcal{O}\left(N^{-1 / 2}\right)$ in (47) gives the same result except the bound at (12)-entry above may be relaxed to $\mathcal{O}\left(N^{-1}\right)$. Then by Lemma 10 we have

$$
R_{2}(z)=N^{\frac{c}{2} \sigma_{3}}\left(I+\left[\begin{array}{cc}
\mathcal{O}\left(\frac{1}{N}\right) & \mathcal{O}\left(\frac{1}{N}\right) \\
\mathcal{O}\left(\frac{1}{\sqrt{N}}\right) & \mathcal{O}\left(\frac{1}{\sqrt{N}}\right)
\end{array}\right]\right) N^{-\frac{c}{2} \sigma_{3}}
$$

Using $R_{1}=\mathcal{R}$ with (34) we get

$$
R_{2} R_{1}=N^{\frac{c}{2} \sigma_{3}}\left(I+\left[\begin{array}{cc}
\mathcal{O}\left(\frac{1}{N}\right) & \frac{\sqrt{2 \pi}\left(a^{2}-1\right)^{c}}{\sqrt{N} a \Gamma(c)} \frac{1}{z-\beta}+\mathcal{O}\left(\frac{1}{N}\right) \\
\mathcal{O}\left(\frac{1}{\sqrt{N}}\right) & \mathcal{O}\left(\frac{1}{\sqrt{N}}\right)
\end{array}\right]\right) N^{-\frac{c}{2} \sigma_{3}} .
$$

From (29) a further decompositions of $\mathcal{F}$ gives $F_{k}=I+\mathcal{O}\left(\zeta^{-3}\right)$ for $k \geq 3$. Then, by Corollary 1, we get

$$
R_{k} \cdots R_{3}=N^{\frac{c}{2} \sigma_{3}}\left(I+\mathcal{O}\left(N^{-3 / 2}\right)\right) N^{-\frac{c}{2} \sigma_{3}}
$$

and

$$
R_{k} \cdots R_{1}=N^{\frac{c}{2} \sigma_{3}}\left(I+\left[\begin{array}{cc}
\mathcal{O}\left(\frac{1}{N}\right) & \frac{\sqrt{2 \pi}\left(a^{2}-1\right)^{c}}{\sqrt{N} a \Gamma(c)} \frac{1}{z-\beta}+\mathcal{O}\left(\frac{1}{N}\right) \\
\mathcal{O}\left(\frac{1}{\sqrt{N}}\right) & \mathcal{O}\left(\frac{1}{\sqrt{N}}\right)
\end{array}\right]\right) N^{-\frac{c}{2} \sigma_{3}}, \quad z \in \partial D_{\beta}
$$

Using Lemma 9, we can have $\widehat{\mathcal{F}}(\zeta)=I+\mathcal{O}\left(\zeta^{-L}\right)$ for an arbitrary $L$. Using Lemma 11 with

$$
\mathcal{R}=R_{k} \cdots R_{1} \quad \text { and } \quad H=H_{k}=I+\mathcal{O}\left(N^{-1 / 2}\right),
$$

we get $Z_{+}^{\infty}\left(Z_{-}^{\infty}\right)^{-1}=I+\mathcal{O}\left(N^{-L}\right)$ on $\partial D_{\beta}$. From the similar argument as in the proof of Theorem 4 , we obtain

$$
Y(z)=e^{\frac{N \ell}{2} \sigma_{3}}\left(I+\mathcal{O}\left(\frac{1}{N^{L}}\right)\right) Z^{\infty}(z)\left[-\star\left(\frac{z}{z-a}\right)^{c} e^{N \phi(z)} \quad 1\right] e^{\frac{-N \ell}{2} \sigma_{3}} e^{N g(z) \sigma_{3}}
$$

uniformly over a compact set for an arbitrary positive integer $L$. The proof is finished by calculations similar to (40) and (41). 


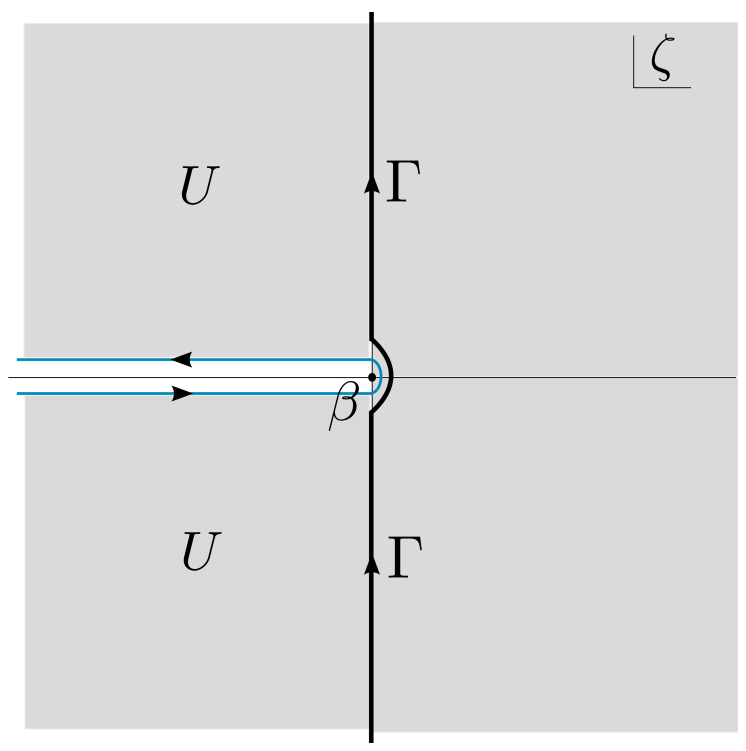

Figure 10: Jump contours of $\mathcal{P}(50)$ in $D_{\beta}$ (left); the shaded region (everywhere except the negative real axis) is $U$.

\section{$6 \quad a<1$}

In this section, we consider the case $a<1$ following closely the analysis of previous two sections for the case $a>1$.

From (7), we obtain

$$
\phi_{A}(z)=\frac{a^{2}-1}{a}(z-\beta)(1+\mathcal{O}(z-\beta)) .
$$

We define $\zeta: D_{\beta} \rightarrow \mathbb{C}$ by (8) where $D_{\beta}$ is a sufficiently small but fixed disc around $z=\beta$ such that $\zeta$ is one-to-one. Under the mapping $\zeta$ the contour $\mathcal{S}$ maps to the imaginary axis.

Inside $D_{\beta}$ we want to find $\mathcal{P}$ such that

$$
Z^{\infty}(z)=\Phi(z)\left(\frac{z-a}{z}\right)^{\frac{c}{2} \sigma_{3}} \mathcal{P}(z)\left(\frac{z-a}{z}\right)^{-\frac{c}{2} \sigma_{3}}
$$

satisfies the jump conditions of $Z$ in (18), i.e.,

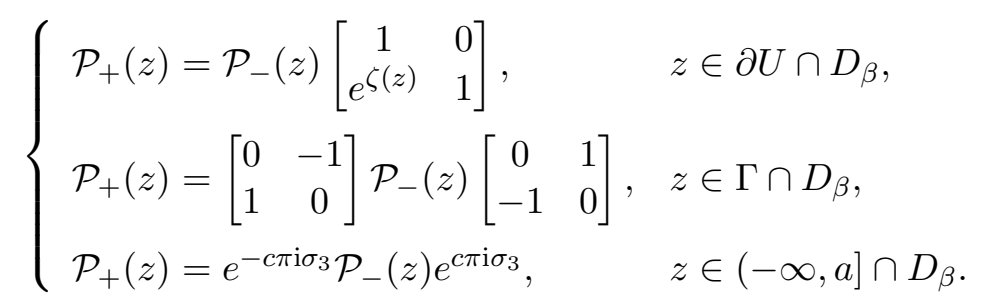


Let us define $S$ by

$$
S=S(\zeta)= \begin{cases}I, & |\arg \zeta|<\pi / 2 \\
{\left[\begin{array}{cc}
0 & 1 \\
-1 & 0
\end{array}\right],} & \text { otherwise }\end{cases}
$$

Here we choose $S$ such that $S^{-1} \zeta(z)^{\frac{c}{2} \sigma_{3}}$ satisfies the left jump of $\mathcal{P}(z)$ from the second and the third equations of (50). Then the matrix function,

$$
W(z)=\zeta(z)^{-\frac{c}{2} \sigma_{3}} S \mathcal{P}(z) S^{-1} \zeta(z)^{\frac{c}{2} \sigma_{3}}
$$

satisfies

$$
W_{+}(z)=W_{-}(z)\left[\begin{array}{cc}
1 & -\zeta(z)^{-c} e^{\zeta(z)} \\
0 & 1
\end{array}\right], \quad z \in \partial U \cap D_{\beta}
$$

Let $H$ be a holomorphic matrix (that will be determined soon). A solution to the above jump condition can be written by $W(z)=H(z) \mathcal{F}(\zeta(z))$ where

$$
\mathcal{F}(\zeta):=\left[\begin{array}{cc}
1 & \frac{-1}{2 \mathrm{i} \pi} \int_{\mathcal{L}} \frac{e^{s}}{s^{c}(s-\zeta)} d s \\
0 & 1
\end{array}\right] .
$$

Here the contour $\mathcal{L}$ is the image of $\partial U$ under $\zeta$, and it begins at $-\infty$, circles the origin once in the counterclockwise direction, and returns to $-\infty$.

Lemma 12. For $z \in D_{\beta}$ we have

$$
\Phi(z)\left(\frac{z-a}{z}\right)^{\frac{c}{2} \sigma_{3}} S^{-1} \zeta(z)^{\frac{c}{2} \sigma_{3}}=\left(N^{c / 2} \eta(z)\right)^{\sigma_{3}}
$$

where $\eta: D_{\beta} \rightarrow \mathbb{C}$,

$$
\eta(z):=\frac{1}{N^{c / 2}}\left(\frac{z \zeta(z)}{z-\beta}\right)^{c / 2},
$$

is a nonvanishing $N$-independent analytic function in $D_{\beta}$.

By Lemma 12, (51) and $W=H \mathcal{F}$ we get

$$
\begin{aligned}
& \Phi(z)\left(\frac{z-a}{z}\right)^{\frac{c}{2} \sigma_{3}} \mathcal{P}(z)\left(\frac{z-a}{z}\right)^{-\frac{c}{2} \sigma_{3}} \\
& =\Phi(z)\left(\frac{z-a}{z}\right)^{\frac{c}{2} \sigma_{3}} S^{-1} \zeta^{(c / 2) \sigma_{3}} W(z) \zeta^{-(c / 2) \sigma_{3}} S\left(\frac{z-a}{z}\right)^{-\frac{c}{2} \sigma_{3}} \\
& =\Phi(z)\left(\frac{z-a}{z}\right)^{\frac{c}{2} \sigma_{3}} S^{-1} \zeta^{(c / 2) \sigma_{3}} H(z) \mathcal{F}(\zeta(z)) \zeta^{-(c / 2) \sigma_{3}} S\left(\frac{z-a}{z}\right)^{-\frac{c}{2} \sigma_{3}} \\
& =\left(N^{c / 2} \eta(z)\right)^{\sigma_{3}} H(z) \mathcal{F}(\zeta(z))\left(N^{c / 2} \eta(z)\right)^{-\sigma_{3}} \Phi(z) .
\end{aligned}
$$

This essentially proves the statement in Lemma 8 for $a<1$. 
Lemma 13. As $|\zeta|$ goes to $\infty, \mathcal{F}$ in (52) satisfies

$$
\mathcal{F}(\zeta) F_{1}(\zeta)^{-1}=I+\mathcal{O}\left(\frac{1}{\left|\zeta^{2}\right|}\right)
$$

uniformly over $c \in(-1,2)$ and

$$
\mathcal{F}(\zeta) F_{1}(\zeta)^{-1} \cdots F_{k}(\zeta)^{-1}=I+\mathcal{O}\left(\frac{1}{\left|\zeta^{k+1}\right|}\right)
$$

where

$$
F_{k}(\zeta)=I+\frac{c_{k}}{\zeta^{k}}\left[\begin{array}{ll}
0 & 1 \\
0 & 0
\end{array}\right], \quad c_{k}=\frac{1}{2 \mathrm{i} \pi} \int_{\mathcal{L}} \frac{s^{k-1} e^{s}}{s^{c}} d s=\frac{\sin (c \pi) \Gamma(k-c)}{\pi(-1)^{k-1}} .
$$

Proof. We only show the proof of (54) as the proof of (55) is similar. The only nonzero entry of $\left(\mathcal{F} F_{1}^{-1}-I\right)$ is the $(12)-$ entry. For $\arg |\zeta|<\pi / 2$, we have

$$
\begin{aligned}
\left|\left(\mathcal{F}(\zeta) F_{1}(\zeta)^{-1}\right)_{12}\right| & =\frac{1}{2 \pi}\left|\int_{\mathcal{L}} \frac{e^{s}}{s^{c}(s-\zeta(z))} d s+\int_{\mathcal{L}} \frac{e^{s}}{s^{c} \zeta(z)} d s\right| \\
& \leq \frac{1}{2 \pi} \int_{\mathcal{L}}\left|\frac{e^{s} s}{s^{c}(\zeta(z)-s) \zeta(z)}\right||d s| \leq \frac{1}{2 \pi} \int_{\mathcal{L}}\left|\frac{e^{s} s}{s^{c} \zeta^{2}}\right||d s| \\
& =\frac{1}{2 \pi\left|\zeta^{2}\right|} \int_{\mathcal{L}}\left|\frac{e^{s} s}{s^{c}}\right||d s| .
\end{aligned}
$$

In the second inequality, we use $|\zeta-s| \geq|\zeta|$ for $\operatorname{Re} \zeta>0$ and $s \in(-\infty, 0]$. One can prove that the last integral is finite by deforming the contour away from the origin so that the integrant is bounded from above.

When $|\arg \zeta| \geq \pi / 2$ a similar argument using the deformation of integration contour leads to the proof of the lemma. Note that the branch cut $(-\infty, 0)$ of $s^{c}$ and the integration contour $\mathcal{L}$ can be deformed, respectively, into $\left\{t e^{\mathrm{i} \theta_{0}}\right\}_{0<t<\infty}$ for $\pi / 2 \leq\left|\theta_{0}\right| \leq \pi$ and the corresponding contour around the new branch cut. We skipped the further details.

Theorem 5. For $a<1$ we get

$$
P_{N}(z)= \begin{cases}z^{N}\left(\frac{z}{z-a}\right)^{c}\left(1+\mathcal{O}\left(\frac{1}{N^{2-c}}\right)\right), & z \in \operatorname{Ext} \mathcal{S} \backslash\left(U \cup D_{\beta}\right), \\ z^{N}\left(\left(\frac{z}{z-a}\right)^{c}-\frac{a\left(1-a^{2}\right)^{c-1}}{N^{1-c} \Gamma(c)} \frac{e^{N \phi_{A}(z)}}{(z-a)}+\mathcal{O}\left(\frac{1}{N^{2-c}}, \frac{e^{N \phi_{A}}}{N^{2-c}}\right)\right), & z \in U \backslash D_{\beta}, \\ z^{N}\left(\left(\frac{z}{z-a}\right)^{c}-\left(\frac{z \zeta(z)}{z-a}\right)^{c} \frac{1}{e^{\zeta(z)}}\left(\hat{f}(\zeta(z))+\mathcal{O}\left(\frac{c}{N}\right)\right)+\mathcal{O}\left(\frac{1}{N^{2-c}}\right)\right), & z \in D_{\beta} .\end{cases}
$$

where

$$
\hat{f}(\zeta)=\frac{-1}{2 \mathrm{i} \pi} \int_{\mathcal{L}} \frac{e^{s}}{s^{c}(s-\zeta)} d s
$$

Here the contour $\mathcal{L}$ is the image of $\partial U$ under $\zeta$, and it begins at $-\infty$, circles the origin once in the counterclockwise direction, and returns to $-\infty$. The error bounds are uniform over $-1<c<2$. 
Proof. From $F_{1}$ in (56) one can obtain $R_{1}$ using Lemma 10 and obtain $H_{1}$ by (49):

$$
\begin{aligned}
& R_{1}(z)=I+\frac{a\left(1-a^{2}\right)^{c-1}}{N^{1-c} \Gamma(c)} \frac{1}{z-a}\left[\begin{array}{ll}
0 & 1 \\
0 & 0
\end{array}\right] \\
& H_{1}(z)=\left(N^{c / 2} \eta(z)\right)^{-\sigma_{3}} R_{1}(z)\left(N^{c / 2} \eta(z)\right)^{\sigma_{3}} F_{1}(\zeta(z))^{-1}=\left[\begin{array}{cc}
1 & h(z) \\
0 & 1
\end{array}\right]
\end{aligned}
$$

where (using $c_{1}=1 / \Gamma(c)$ that appears in $F_{1}$ )

$$
h(z)=\left(\frac{z-a}{z \zeta(z)}\right)^{c}\left(\frac{a\left(1-a^{2}\right)^{c-1}}{N^{1-c} \Gamma(c)} \frac{1}{z-a}\right)-\frac{1}{\zeta(z) \Gamma(c)}=\mathcal{O}\left(\frac{c}{N}\right) .
$$

Setting $\mathcal{R}=R_{1}$ and $H=H_{1}$, we can define $Z^{\infty}$ by (33) and (36). Defining the error matrix by $\mathcal{E}=Z^{\infty} Z^{-1}$, by the similar calculation as (38) with $\widehat{\mathcal{F}}=\mathcal{F} F_{1}^{-1}$ and (54), we get

$$
\mathcal{E}_{+}(z) \mathcal{E}_{-}^{-1}(z)=I+\mathcal{O}\left(\frac{1}{N^{2-c}}\right), \quad z \in \partial D_{\beta}
$$

uniformly over $c \in(-1,2)$. By the same argument as in the proof of Theorem 4 we obtain

$$
Z(z)=\left(I+\mathcal{O}\left(\frac{1}{N^{2-c}}\right)\right) Z^{\infty}(z)
$$

The proof is finished by the calculations exactly similar to (40) and (41). To add a little more detail, inside $D_{\beta}$ we need to use (58) to obtain the final result. Below we write the strong asymptotics before using (58) as an example.

$$
\left(\left(\frac{z}{z-a}\right)^{c}-\left(\frac{z \zeta(z)}{z-a}\right)^{c}(\hat{f}(\zeta(z))+h(z)) e^{N \phi(z)}+\mathcal{O}\left(\frac{1}{N^{2-c}}\right)\right) e^{N g(z)}, \quad z \in \operatorname{Ext} \mathcal{S} \cap D_{\beta} .
$$

We skip the computation.

Proof of Theorem 3. The proof will be similar to the above proof and the proof of Theorem 2,

By (56), (57) and (43) we get

$$
\begin{aligned}
\widetilde{F}_{2}(z) & =\left(N^{\frac{c}{2}} \eta(z)\right)^{\sigma_{3}} H_{1}(z) F_{2}(\zeta(z)) H_{1}^{-1}(z)\left(N^{\frac{c}{2}} \eta(z)\right)^{-\sigma_{3}} \\
& =N^{\frac{c}{2} \sigma_{3}}\left(I+\left[\begin{array}{cc}
0 & \mathcal{O}\left(N^{-2}\right) \\
0 & 0
\end{array}\right]\right) N^{-\frac{c}{2} \sigma_{3}}, \quad z \in \partial D_{\beta} .
\end{aligned}
$$

From Lemma 10 and (59) we have

$$
R_{2}(z)=N^{\frac{c}{2} \sigma_{3}}\left(I+\left[\begin{array}{cc}
0 & \mathcal{O}\left(N^{-2}\right) \\
0 & 0
\end{array}\right]\right) N^{-\frac{c}{2} \sigma_{3}} .
$$

Combined with $R_{1}$ in (57), we get

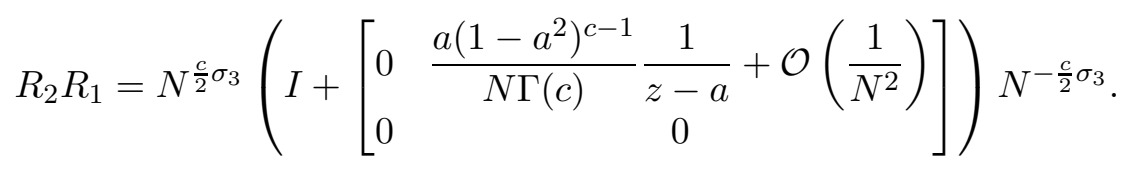


From (56) in Lemma 13, we have $F_{k}=I+\mathcal{O}\left(\zeta^{-3}\right)$ for $k \geq 3$. By Corollary 11, we obtain

$$
R_{k} \cdots R_{3}=N^{\frac{c}{2} \sigma_{3}}\left(I+\mathcal{O}\left(N^{-3}\right)\right) N^{-\frac{c}{2} \sigma_{3}} .
$$

In fact, following the inductive construction of $R_{k}$ and $H_{k}$ in Section 5 , one can find that $R_{k}$ 's are all upper diagonal matrix. Therefore, we get

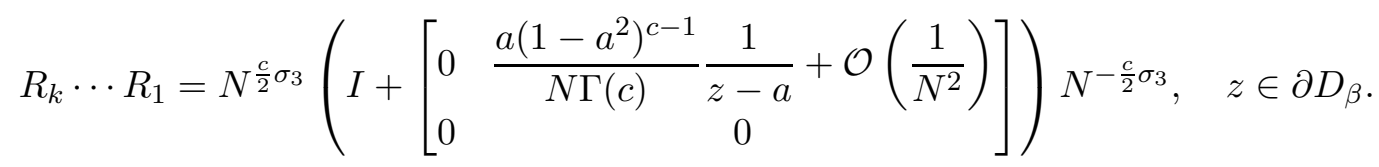

Using Lemma 9, we can have $\widehat{\mathcal{F}}(\zeta)=I+\mathcal{O}\left(\zeta^{-L}\right)$ for an arbitrary L. Using Lemma 11 with

$$
\mathcal{R}=R_{k} \cdots R_{1} \quad \text { and } \quad H=H_{k}=I+\mathcal{O}\left(N^{-1}\right),
$$

we get $Z_{+}^{\infty}\left(Z_{-}^{\infty}\right)^{-1}=I+\mathcal{O}\left(N^{-L}\right)$ on $\partial D_{\beta}$. From the similar argument as in the proof of Theorem 4 , we obtain

$$
Y(z)=e^{\frac{N \ell}{2} \sigma_{3}}\left(I+\mathcal{O}\left(\frac{1}{N^{L}}\right)\right) Z^{\infty}(z)\left[-\star\left(\frac{z}{z-a}\right)^{c} e^{N \phi(z)} \quad 1\right] e^{\frac{-N \ell}{2} \sigma_{3}} e^{N g(z) \sigma_{3}}
$$

for an arbitrary positive integer $L$. The proof is finished by calculations similar to (40) and (41).

\section{Critical case: $a=1$}

In this section we consider $a=1+\mathcal{O}(1 / \sqrt{N})$. Here we only argue that the strong asymptotics can be obtained through the parametrix of Painlevé IV equation (as suggested in [2]) following the similar steps described previously.

There is a disk $D_{1}$ centered at 1 such that there exists a univalent map $\zeta: D_{1} \rightarrow \mathbb{C}$ that satisfies

$$
(\zeta(z)+x)^{2}=N \phi_{A}(z)-N \phi_{A}(1 / a)
$$

where

$$
x:=\sqrt{N \phi_{A}(a)-N \phi_{A}(1 / a)}=\sqrt{2 N}(a-1)(1+\mathcal{O}(a-1)) .
$$

Under the mapping $\zeta$, we have $\zeta(a)=0$ and the critical point of $\phi_{A}$ maps to $-x$; note that $\phi(1 / a)$ is the critical value of $\phi_{A}$.

Inside $D_{1}$ we require that $\Phi(z)\left(\frac{z-a}{z}\right)^{\frac{c}{2} \sigma_{3}} \mathcal{P}(z)\left(\frac{z-a}{z}\right)^{-\frac{c}{2} \sigma_{3}}$ satisfies the jump conditions (18) of $Z$. With the boundary condition of $\mathcal{P}$ on $\partial D_{1}$ this leads to the following jumps of $\mathcal{P}$ inside $D_{1}$ :

$$
\begin{cases}\mathcal{P}_{+}(z)=\mathcal{P}_{-}(z)\left[\begin{array}{cc}
1 & 0 \\
e^{-N \phi_{A}(z)} & 1
\end{array}\right], & z \in \partial U \cap \operatorname{Int} \Gamma, \\
\mathcal{P}_{+}(z)=\mathcal{P}_{-}(z)\left[\begin{array}{cc}
1 & 0 \\
e^{N \phi_{A}(z)} & 1
\end{array}\right], & z \in \partial U \cap \operatorname{Ext} \Gamma, \\
\mathcal{P}_{+}(z)=\left[\begin{array}{cc}
0 & -1 \\
1 & 0
\end{array}\right] \mathcal{P}_{-}(z)\left[\begin{array}{cc}
0 & 1 \\
-1 & 0
\end{array}\right], & z \in \Gamma \cap U, \\
\mathcal{P}_{+}(z)=e^{-c \pi \mathrm{i} \sigma_{3}} \mathcal{P}_{-}(z) e^{c \pi \mathrm{i} \sigma_{3}}, & z \in(0, a], \\
\mathcal{P}(z)=I+o(1), & z \in \partial D_{1} .\end{cases}
$$




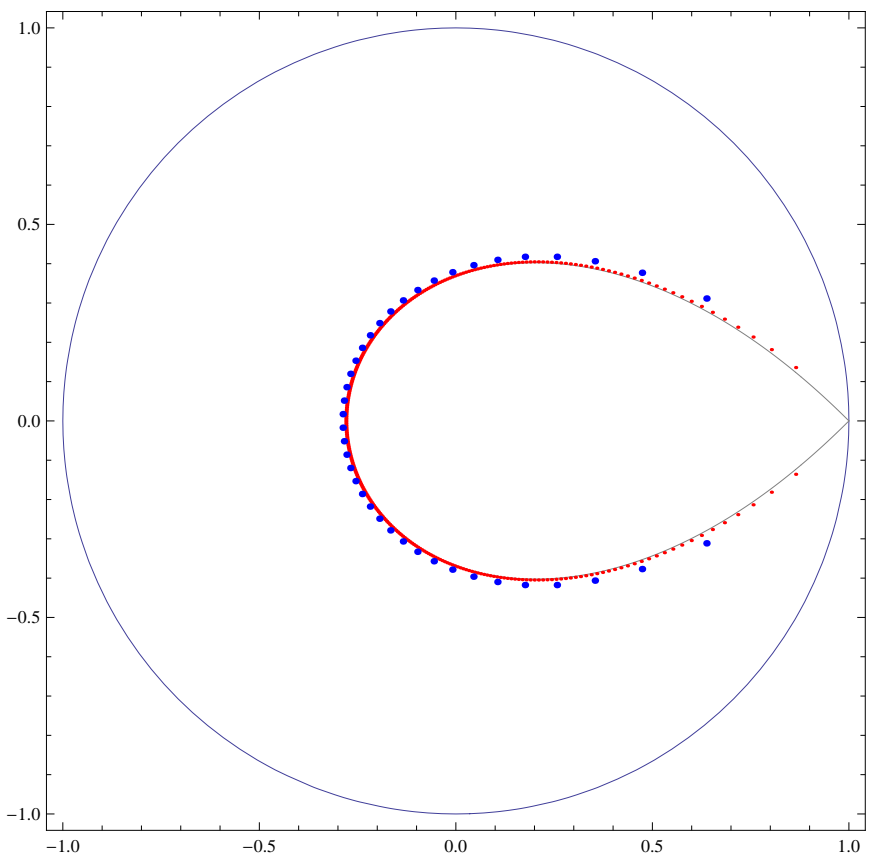

Figure 11: The zeros of orthogonal polynomials with degrees 40 (blue) and 300 (red), $c=1$ and $a=1$. The solid line inside the disk is $\mathcal{S}$.

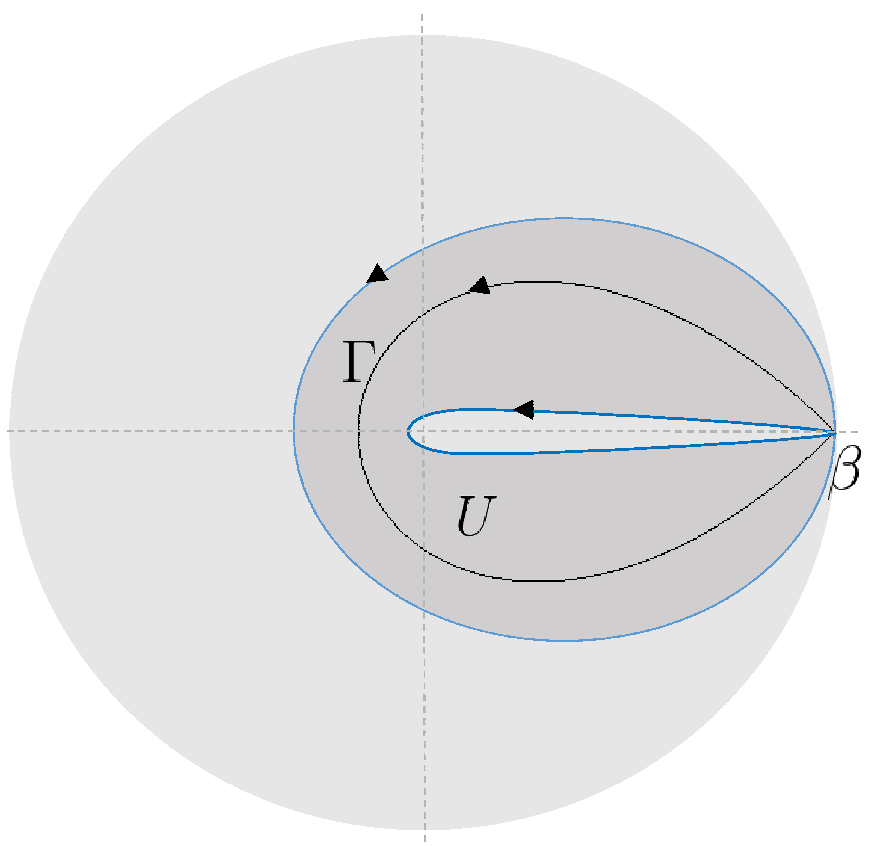

Figure 12: Contours for the Riemann-Hilbert problem of $\Phi$ when $a \approx 1$. $\Gamma$ is the black curves and $U$ is the shaded region bounded by the blue curves. 
Here $U$ and $\Gamma$ are given similarly by those for $a>1$ except the segment $[\beta, a]$ becomes a point at 1 , see Figure 12. We will show that such $\mathcal{P}$ can be written in terms of the solution of the Painlevé IV equation. To achieve this, we want to transform $\mathcal{P}$ into a new matrix function, $W$, with only constant jump matrices from the right. Such transform is given by

$$
W(z)=e^{-\frac{\ell_{x}}{2} \sigma_{3}} \zeta(z)^{\frac{c}{2} \sigma_{3}} S \cdot \mathcal{P}(z) \cdot T(z)^{-1} S^{-1}, \quad z \in D_{1}
$$

using a diagonal matrices $T$, a piecewise constant matrix $S$ and a constant $\ell_{x}$, defined by

$$
\begin{aligned}
T(z) & =\exp \left(\frac{N}{2}(-1)^{\nu} \phi_{A}(z) \sigma_{3}\right)=\exp \left[\frac{(-1)^{\nu}}{2}\left(\zeta(z)^{2}+2 x \zeta(z)+\ell_{x}\right) \sigma_{3}\right], \\
\ell_{x} & =x^{2}+N \phi_{A}(1 / a), \quad S=S(z)=\left[\begin{array}{ll}
0 & 1 \\
1 & 0
\end{array}\right] \cdot\left[\begin{array}{cc}
0 & 1 \\
-1 & 0
\end{array}\right]^{\nu},
\end{aligned}
$$

where

$$
\nu= \begin{cases}0, & z \in \operatorname{Ext} \Gamma, \\ 1, & z \in \operatorname{Int} \Gamma .\end{cases}
$$

Here we chose $S$ such that $S^{-1} \zeta(z)^{-\frac{c}{2} \sigma_{3}}$ satisfies all the left jumps of $\mathcal{P}$, i.e.,

$$
\begin{aligned}
& \left(S^{-1} \zeta(z)^{-\frac{c}{2} \sigma_{3}}\right)_{+}=\left[\begin{array}{cc}
0 & 1 \\
-1 & 0
\end{array}\right]\left(S^{-1} \zeta(z)^{-\frac{c}{2} \sigma_{3}}\right)_{-}, \quad z \in \Gamma \cap U \\
& \left(S^{-1} \zeta^{-\frac{c}{2} \sigma_{3}}\right)_{+}=e^{-c \pi i \sigma_{3}}\left(S^{-1} \zeta^{-\frac{c}{2} \sigma_{3}}\right)_{-}, \quad z \in[-\infty, 0] .
\end{aligned}
$$

Consequently, $W$ has the jump matrices only from the right. Furthermore, the jump matrices of $W$ are constant matrices because of the right multipliction of $T$ in (60), and the jump on $\Gamma$ disappears by the right multiplication by $S^{-1}$. We obtain the jump condition of $W$ by

$$
W_{+}(z)=W_{-}(z) \begin{cases}{\left[\begin{array}{cc}
1 & 0 \\
s_{1} & 1
\end{array}\right],} & \zeta(z) \in \mathbb{R}^{+}, \\
{\left[\begin{array}{cc}
1 & s_{2} \\
0 & 1
\end{array}\right],} & \zeta(z) \in \mathbb{i}^{+}, \\
{\left[\begin{array}{cc}
1 & 0 \\
s_{3} & 1
\end{array}\right],} & \zeta(z) \in \mathbb{R}^{-}, \\
{\left[\begin{array}{cc}
1 & s_{4} \\
0 & 1
\end{array}\right],} & \zeta(z) \in \mathbb{R}^{-},\end{cases}
$$

where $s_{1}=0, s_{2}=1, s_{3}=e^{2 \mathrm{i} c \pi}-1$ and $s_{4}=-e^{-2 \mathrm{i} c \pi}$. The boundary condition at $\partial D_{1}$ gives

$$
W(z)=\zeta(z)^{\frac{c}{2} \sigma_{3}}(I+o(1)) e^{\left(\frac{\zeta(z)^{2}}{2}+x \zeta(z)\right) \sigma_{3}}, \quad z \in \partial D_{\beta} .
$$

Here we used that $\ell_{x}=\mathcal{O}(1)$ for $a=1+\mathcal{O}(1 / \sqrt{N})$. According to page 34 of [6] (or [9]) the RiemannHilbert problem for the Painlevé IV parametrix $-\Psi$, following the notation in [6] - exactly satisfies the jump condition above and the boundary condition:

$$
\Psi(\zeta, x)=\left(I+\frac{\Psi_{-1}(x)}{\zeta}+\frac{\Psi_{-2}(x)}{\zeta^{2}}+\mathcal{O}\left(\frac{1}{\zeta^{3}}\right)\right) e^{\left(\frac{\zeta^{2}}{2}+x \zeta\right)} \zeta^{-\Theta_{\infty} \sigma_{3}}, \quad z \rightarrow \infty
$$


when

$$
\left(1+s_{2} s_{3}\right) e^{2 \mathrm{i} \pi \Theta_{\infty}}+\left[s_{1} s_{4}+\left(1+s_{3} s_{4}\right)\left(1+s_{1} s_{2}\right)\right] e^{-2 \mathrm{i} \pi \Theta_{\infty}}=2 \cos 2 \pi \Theta .
$$

In our case we get $\Theta=c / 2, \Theta_{\infty}=-c / 2$. It means that, using the same strategy to Section 4 and 6 , we could get the similar result about the asymptotics of orthogonal polynomial in terms of Painlevé IV equation:

$$
\frac{d^{2} u}{d x^{2}}=\frac{1}{2 u}\left(\frac{d u}{d x}\right)^{2}+\frac{3}{2} u^{3}+4 x u^{2}+\left(2+2 x^{2}-4 \Theta_{\infty}\right) u-\frac{8 \Theta^{2}}{u},
$$

where the solution $u$ is related to the Riemann-Hilbert problem by

$$
u(x)=-2 x-\frac{d}{d x} \log \left(\left(\Psi_{-1}\right)(x)_{12}\right)
$$

\section{A Proof of Lemma 6}

By [19] we can write

$$
D_{-c}(\zeta)=2^{-c / 2} e^{-\zeta^{2} / 4} U\left(\frac{c}{2}, \frac{1}{2}, \frac{\zeta^{2}}{2}\right)
$$

where $U$ has the following asymptotic expansion as $|\zeta| \rightarrow \infty$.

$$
U\left(\frac{c}{2}, \frac{1}{2}, \frac{\zeta^{2}}{2}\right)=\left(\frac{\zeta^{2}}{2}\right)^{-\frac{c}{2}} \sum_{s=0}^{n-1}\left(-\frac{\zeta^{2}}{2}\right)^{-s} \frac{\left(\frac{c}{2}\right)_{s}\left(\frac{c+1}{2}\right)_{s}}{s !\left(2 \zeta^{2}\right)^{s}}+\widehat{\varepsilon_{n}}\left(\frac{\zeta^{2}}{2}\right), \quad|\arg \zeta|<\frac{\pi}{2} .
$$

The error term $\widehat{\varepsilon_{n}}$ is bounded by

$$
\left|\widehat{\varepsilon_{n}}\left(\frac{\zeta^{2}}{2}\right)\right| \leq 2^{\frac{c}{2}+n+1} \alpha\left|\frac{\left(\frac{c}{2}\right)_{n}\left(\frac{c+1}{2}\right)_{n}}{n !\left(\zeta^{2}\right)^{n+\frac{c}{2}}}\right| \exp \left(\frac{4 \alpha \rho}{\left|\zeta^{2}\right|}\right)
$$

where

$$
\alpha=\frac{1}{1-\sigma}, \quad \sigma=\left|\frac{1-2 c}{\zeta^{2}}\right|, \quad \rho=\left|\frac{c^{2}-c+1}{4}\right|+\frac{\sigma\left(1+\frac{\sigma}{4}\right)}{(1-\sigma)^{2}}
$$

We have

$$
\left|\varepsilon_{n}(\zeta)\right|=2^{-\frac{c}{2}}|\zeta|^{c}\left|\widehat{\varepsilon_{n}}\left(\frac{\zeta^{2}}{2}\right)\right| \leq C\left|\frac{\left(\frac{c}{2}\right)_{n}\left(\frac{c+1}{2}\right)_{n}}{n !\left(\zeta^{2}\right)^{n}}\right|
$$

where

$$
C=\frac{2^{n+1}\left|\zeta^{2}\right|}{\left(\left|\zeta^{2}\right|-|1-2 c|\right)} \exp \left(\left|\frac{c^{2}-c+1}{4\left(\left|\zeta^{2}\right|-|1-2 c|\right)}\right|+\frac{|1-2 c|\left(\left|\zeta^{2}\right|+\frac{|1-2 c|}{4}\right)}{\left(\left|\zeta^{2}\right|-|1-2 c|\right)^{3}}\right) .
$$

For $\left|\zeta^{2}\right| /|1-2 c|$ big enough, we have $C \leq 2^{n+2}$. 


\section{B Lax pair: how the numerical calculation is done}

Define $\tilde{Y}(z)$ by $\tilde{Y}(z)=\widetilde{Y}_{n}(z)=Y(z)\left[\begin{array}{cc}\left(\frac{z-a}{z}\right)^{c} \frac{1}{e^{N a z}} & 0 \\ 0 & z^{n}\end{array}\right]$, then the Riemann-Hilbert problem for $\tilde{Y}(z)$ is

$$
\begin{cases}\tilde{Y}(z) \text { is holomorphic in } \mathbb{C} \backslash \Gamma, & \\
\widetilde{Y}_{+}(z)=\widetilde{Y}_{-}(z)\left[\begin{array}{ll}
1 & 1 \\
0 & 1
\end{array}\right], & z \in \Gamma, \\
\tilde{Y}_{+}(z)=\tilde{Y}_{-}(z)\left[\begin{array}{cc}
e^{2 c \pi \mathrm{i}} & 0 \\
0 & 1
\end{array}\right], & z \in(0, a), \\
\widetilde{Y}(z)=\left(I+\mathcal{O}\left(\frac{1}{z}\right)\right)\left[\begin{array}{cc}
\left(\frac{z-a}{z}\right)^{c} \frac{z^{n}}{e^{N a z}} & 0 \\
0 & 1
\end{array}\right], & z \rightarrow \infty .\end{cases}
$$

We observe $\tilde{Y}_{n}(z)$ and $\widetilde{Y}_{n+1}(z)$ have the same jump matrices. Since $\operatorname{det} Y(z) \equiv 1$, the inverse of $\tilde{Y}(z)$ exists in $\mathbb{C} \backslash(\Gamma \cup(0, a))$, and we can define

$$
A_{n}(z)=\frac{d \widetilde{Y}_{n}(z)}{d z} \widetilde{Y}_{n}(z)^{-1}
$$

The matrix function $A_{n}(z)$ is meromorphic and can be determined by identifying the singularities. For $z \rightarrow \infty$, writing (we know that $c_{n}$ below is not related to the charge " $c$ " in the potential)

$$
\widetilde{Y}_{n}(z)=\left(I+\frac{1}{z}\left[\begin{array}{cc}
a_{n} & b_{n} \\
c_{n} & d_{n}
\end{array}\right]+\cdots\right)\left[\begin{array}{cc}
\left(\frac{z-a}{z}\right)^{c} \frac{z^{n}}{e^{N a z}} & 0 \\
0 & 1
\end{array}\right]
$$

we get

$$
A_{n}(z)=\left[\begin{array}{cc}
-N a & 0 \\
0 & 0
\end{array}\right]+\frac{1}{z}\left[\begin{array}{cc}
n & N a b_{n} \\
-N a c_{n} & 0
\end{array}\right]+\mathcal{O}\left(z^{-2}\right)
$$

Similarly we get the following for $z \rightarrow 0$.

$$
\begin{aligned}
& \tilde{Y}_{n}(z)=\left[\begin{array}{ll}
\alpha_{n} & \beta_{n} \\
\gamma_{n} & \eta_{n}
\end{array}\right](I+\mathcal{O}(z))\left[\begin{array}{cc}
\left(\frac{z-a}{z}\right)^{c} \frac{1}{e^{N a z}} & 0 \\
0 & z^{n}
\end{array}\right] \\
& A_{n}(z)=\frac{1}{z}\left[\begin{array}{cc}
-c-(c+n) \beta_{n} \gamma_{n} & (c+n) \alpha_{n} \beta_{n} \\
-(c+n) \gamma_{n} \eta_{n} & n+(c+n) \beta_{n} \gamma_{n}
\end{array}\right] .
\end{aligned}
$$

Therefore, we obtain

$$
\begin{aligned}
A_{n}(z) & =\left[\begin{array}{cc}
-N a & 0 \\
0 & 0
\end{array}\right]+\frac{1}{z}\left[\begin{array}{cc}
-c-(c+n) \beta_{n} \gamma_{n} & (c+n) \alpha_{n} \beta_{n} \\
-(c+n) \gamma_{n} \eta_{n} & n+(c+n) \beta_{n} \gamma_{n}
\end{array}\right] \\
& +\frac{1}{z-a}\left[\begin{array}{cc}
(c+n)\left(1+\beta_{n} \gamma_{n}\right) & N a b_{n}-(c+n) \alpha_{n} \beta_{n} \\
-N a c_{n}+(c+n) \gamma_{n} \eta_{n} & -n-(c+n) \beta_{n} \gamma_{n}
\end{array}\right] .
\end{aligned}
$$

Defining $M_{n}(z)=\widetilde{Y}_{n+1}(z) \widetilde{Y}_{n}(z)^{-1}$ we obtain, by the similar procedure as above,

$$
M_{n}(z)=\left[\begin{array}{cc}
z+a_{n+1}-a_{n} & -b_{n} \\
c_{n+1} & 1
\end{array}\right] \text {. }
$$


The compatibility of the Lax pair,

$$
\begin{gathered}
\frac{d \widetilde{Y}_{n}(z)}{d z}=A_{n}(z) \widetilde{Y}_{n}(z), \\
\widetilde{Y}_{n+1}(z)=M_{n}(z) \widetilde{Y}_{n}(z),
\end{gathered}
$$

gives

$$
A_{n+1}(z) M_{n}(z)=\frac{d M_{n}(z)}{d z}+M_{n}(z) A_{n}(z)
$$

This gives the following recurrence relation:

$$
\begin{aligned}
& a_{n+1}=a_{n}+\frac{b_{n}\left(1+\beta_{n} \gamma_{n}\right)}{\alpha_{n} \beta_{n}}, \quad \alpha_{n+1}=\frac{b_{n}}{\beta_{n}}, \quad \gamma_{n+1}=-\frac{1}{\beta_{n}}, \\
& b_{n+1}=\frac{\left(1+n+a^{2} N\right) b_{n}}{a N}+\frac{(c+n) \alpha_{n} \beta_{n}}{N}+\frac{b_{n}^{2}\left(1+\beta_{n} \gamma_{n}\right)}{\alpha_{n} \beta_{n}}, \\
& \beta_{n+1}=\frac{\tilde{c}}{(1+c+n)\left((c+n) \alpha_{n} \beta_{n}-a N b_{n}\right) \alpha_{n}^{2} \beta_{n}},
\end{aligned}
$$

where

$$
\begin{aligned}
& \tilde{c}=a^{2} N-c-a(1+2(c+n)) \alpha_{n} \beta_{n}+\left(a^{2} N-c-a(c+n) \alpha_{n} \beta_{n}\right) \beta_{n} \gamma_{n} \\
& \quad+(c+n)(c+n+1) \alpha_{n}^{3} \beta_{n}^{3}+a N^{2} b_{n}^{3}\left(1+\beta_{n} \gamma_{n}\right)^{2} \\
& a_{0}=0, \quad b_{0}=a, \quad \alpha_{0}=1, \quad \beta_{0}=1+a^{2} N, \quad \gamma_{0}=0 .
\end{aligned}
$$

The last line contains the initial condition of the recurrence relation. We used the above relation to generate the orthogonal polynomials numerically.

\section{References}

[1] Y. Ameur, H. Hedenmalm, N. Makarov, Fluctuations of Eigenvalues of Random Normal Matrices, Duke Math. J., Vol.159, No.1:31-81, (2011).

[2] F. Balogh, T. Grava, D. Merzi, Orthogonal polynomials for a class of measures with discrete rotational symmetries in the complex plane, (arXiv:1509.05331 v1), (2015).

[3] F. Balogh, M. Bertola, S.-Y. Lee, and K.T.-R. Mclaughlin. Strong asymptotics of the orthogonal polynomials with respect to a measure supported on the plane, Communications of Pure and Applied Mathematics, 68(1):112-172, (2015).

[4] M. Bertola and S.-Y. Lee. First colonization of a spectral outpost in random matrix theory. Constr. Approx.,30(2), 225-263, (2008).

[5] P.M. Bleher, A. Kuijlaars, Orthogonal polynomials in the normal matrix model with a cubic potential, Adv. Math. 230, 1272-1321, (2012).

[6] D. Dai, A. Kuijlaars, Painleve IV asymptotics for orthogonal polynomials with respect to a modified Laguerre weight, Stud. Appl. Math. 122 , 29-83, (2009). 
[7] P.A. Deift. Orthogonal polynomials and random matrices: a Riemann-Hilbert approach, volume 3 of Courant Lecture Notes in Mathematics. New York University Courant Institute of Mathematical Sciences, New York, (1999).

[8] P. Deift, T. Kriecherbauer, K. T-R McLaughlin, S. Venakides, and X. Zhou. Strong asymptotics of orthogonal polynomials with respect to exponential weights. Comm. Pure Appl. Math., 52(12):1491$1552,(1999)$.

[9] A.S. Fokas, A.R. Its, A.A. Kapaev, and V.Y. Novokshenov, Painleve Transcendents, The RiemannHilbert Approach: Mathematical survey and Monographs, Vol. 128, Amer. Math. Soc., Providence, RI, (2006).

[10] B. Gustafsson, On Mother Bodies of Convex Polyhedra, SIAM J. Math. Anal., Vol.29,No.5:11061117, (1998).

[11] B. Gustafsson, M. Sakai, On Potential Theoretic Skeletons of Polyhedra, Geom. Dedicata 76, 1-30, (1999).

[12] H. Hedenmalm, N. Makarov, Coulomb gas ensembles and Laplacian growth, Proc. London Math. Soc. 106 (4): 859-907 doi: 10.1112/plms/pds032, (2013).

[13] A. Kuijlaars, K. T-R Mclaughlin, Asymptotic zero behavior of Laguerre polynomial with negative parameter, J. Constr. Approx. 20 497-523, (2004)

[14] A. Kuijlaars, G.L.F. Silva, S-curves in polynomial external fields, J. Approx. Theory 191, 1-37, (2015)

[15] A. Kuijlaars, A. Lopez, The normal matrix model with a monomial potential, a vector equilibrium problem, and multiple orthogonal polynomials on a star, Nonlinearity 28, 347-406, (2015).

[16] A. Kuijlaars, A. Tovbis, The supercritical regime in the normal matrix model with cubic potential, Adv. Math. 283 , 530-587, (2015).

[17] H. N. Mhaskar, E. B. Saff, The distribution of zeros of asymptotically extremal polynomials , J. Approx. Theory 65, no. 3, 279-300, (1991).

[18] E. B. Saff, V. Totik, Logarithmic potentials with external fields, Grundlehren der mathematischen Wissenschaften, 316, Springer, Berlin, (1997).

[19] F.W.J. Olver, D.W. Lozier, R.F. Boisvert, C.W. Clark, NIST Handbook of Mathematical Functions, NIST and Cambridge University Press, (2010).

[20] E.T. Whittaker, G.N. Watson, A Course of Modern Analysis, Cambridge University Press, Chapter 16, Part 5, (1996). 